\title{
Visualizing translocation dynamics and nascent transcript errors in paused RNA polymerases in vivo
}

\author{
Masahiko Imashimizu', Hiroki Takahashi ${ }^{2}$, Taku Oshima ${ }^{3}$, Carl McIntosh' ${ }^{1}$, Mikhail Bubunenko ${ }^{1}$, Donald L. Court ${ }^{1}$ \\ and Mikhail Kashlev ${ }^{1 *}$
}

\begin{abstract}
Background: Transcription elongation is frequently interrupted by pausing signals in DNA, with downstream effects on gene expression. Transcription errors also induce prolonged pausing, which can lead to a destabilized genome by interfering with DNA replication. Mechanisms of pausing associated with translocation blocks and misincorporation have been characterized in vitro, but not in vivo.

Results: We investigate the pausing pattern of RNA polymerase (RNAP) in Escherichia coli by a novel approach, combining native elongating transcript sequencing (NET-seq) with RNase footprinting of the transcripts (RNET-seq). We reveal that the G-dC base pair at the $5^{\prime}$ end of the RNA-DNA hybrid interferes with RNAP translocation. The distance between the $5^{\prime} \mathrm{G}-\mathrm{dC}$ base pair and the $3^{\prime}$ end of RNA fluctuates over a three-nucleotide width. Thus, the G-dC base pair can induce pausing in post-translocated, pre-translocated, and backtracked states of RNAP. Additionally, a CpG sequence of the template DNA strand spanning the active site of RNAP inhibits elongation and induces G-to-A errors, which leads to backtracking of RNAP. Gre factors efficiently proofread the errors and rescue the backtracked complexes. We also find that pausing events are enriched in the $5^{\prime}$ untranslated region and antisense transcription of mRNA genes and are reduced in rRNA genes.
\end{abstract}

Conclusions: In E. coli, robust transcriptional pausing involves RNAP interaction with G-dC at the upstream end of the RNA-DNA hybrid, which interferes with translocation. CpG DNA sequences induce transcriptional pausing and G-to-A errors.

\section{Background}

RNA polymerase (RNAP) transcribes DNA of different structural and chemical sequences. Interaction of RNAP with some of these sequences results in transcriptional pausing, which occurs on average every $100 \mathrm{bp}$ of transcribed DNA in vitro [1]. Regulation of elongation via pausing has a variety of physiological consequences [1]. In prokaryotes, the RNAP pausing/anti-pausing system that utilizes $\mathrm{RfaH}$ protein controls expression of genes involved in DNA transfer and virulence [2, 3]. Many regulatory events derived from pausing appear to be localized in promoter-proximal regions in eukaryotes or the $5^{\prime}$ untranslated region (UTR) of mRNA genes in prokaryotes

\footnotetext{
* Correspondence: kashlevm@mail.nih.gov

${ }^{1}$ Center for Cancer Research, National Cancer Institute, Frederick, MD 21702, USA

Full list of author information is available at the end of the article
}

[2, 4-6]. For example, eukaryotic RNAPII tends to pause in a region located $\leq 100$ bp downstream of a transcription start site, and is controlled by accessory protein factors such as NELF/DSIF [4, 7]. These paused polymerases allow a rapid transcription response to environmental stimuli and are used during development in higher eukaryotes $[4,6]$. The RNAPII pausing at promoter-proximal regions in eukaryotes also plays a critical role in protecting these regions from adopting repressive chromatin structures, thereby maintaining an open promoter complex for highly expressed genes $[8,9]$. In prokaryotes, pausing plays a key role in transcription attenuation and termination and in synchronization of transcription and translation $[1,3,10]$.

An elongation complex (EC) consists of RNAP bound to double-stranded DNA and the RNA-DNA hybrid with the 3' end of the RNA positioned in the active
Ciomed Central

(C) 2015 Imashimizu et al. This is an Open Access article distributed under the terms of the Creative Commons Attribution License (http://creativecommons.org/licenses/by/4.0), which permits unrestricted use, distribution, and reproduction in any medium, provided the original work is properly credited. The Creative Commons Public Domain Dedication waiver (http:// creativecommons.org/publicdomain/zero/1.0/) applies to the data made available in this article, unless otherwise stated. 
center of the enzyme [11]. The hybrid length fluctuates between 9-bp and 10-bp length depending on the translocation state of RNAP. After phosphodiester bond formation, the movement of the RNA-DNA hybrid back along the catalytic cleft vacates the active center, enables binding of the next NTP and reduces the length of the RNA-DNA hybrid from 10 to $9 \mathrm{bp}$ in a process called translocation [1]. Translocation is a smooth process except in cases where certain DNA sequences impose an intrinsic translocation barrier $[1,12]$. This block of translocation as well as the inhibition of the bond formation after translocation causes RNAP pausing [1]. Protein factors exist that strengthen or weaken pausing by targeting translocation, such as the archaeal/eukaryotic Spt5 and bacterial NusG/NusA $[3,13,14]$ as well as the Nun/N transcription termination/antitermination proteins of lambdoid phages $[1,15]$. Pausing of EC within the post-translocated or pretranslocated state is enhanced when an RNA hairpin is formed immediately upstream of the hybrid [16, 17].

Some pausing signals in Escherichia coli, such as ops sequence, involve backtracking of RNAP along DNA [18]. Backtracking stabilizes pausing [12, 19] and leads to extrusion of one or more nucleotides of the 3' RNA end beyond the active center [20]. A stably backtracked EC forms a roadblock to DNA replication [21], which can be highly toxic to the cell [22-24]. A direct assessment of transcription fidelity by RNA-seq in vivo and in vitro showed that an error at the 3' end of a nascent RNA causes long transcription pausing by inducing RNAP backtracking [25]. It was also shown that transcription errors cause some heritable phenotypic changes [26, 27], which have been thought to affect aging [28] and carcinogenesis [29, 30]. Bacterial GreA and GreB or eukaryotic TFIIS proteins induce endonucleolytic RNA cleavage of any extruded 3' RNA, with or without errors, thereby allowing renewed transcription in the backtracked EC [31, $32]$, which ensures better fidelity and removes the DNA replication barrier [22-25].

Extensive biochemical and single-molecule experiments have identified the steps involved in pausing in vitro [1]: Pausing can be caused by (i) a misalignment of incoming NTP and complementary template DNA base within the active site of the post-translocated RNAP [33], and (ii) an intrinsic barrier caused by DNA sequence during forward translocation from the pretranslocated state $[13,34]$. This latter type of pausing can be stabilized by backtracking [12]. However, little is known about how broadly these mechanisms for pausing identified in vitro are involved in transcription regulation in vivo.

In the present work, we employed native elongating transcript sequencing (NET-seq) [35] to identify RNAP pause sites and error hotspots in the E. coli chromosome by making an assumption that transcription errors contribute to pausing in vivo. After paused RNAP complexes are isolated from the genome, RNases are used to trim excess RNA from the $5^{\prime}$ ends leaving only the nascent RNA that is protected by RNAP. Thus, RNET-seq stands for RNase footprinting followed by NET-seq. A previous in vitro study showed that an RNAP forming an EC protects different lengths of the 3'-proximal transcript from trimming by RNases A and T1 depending on the EC translocation state [36]. Post-translocated, pretranslocated, and backtracked complexes protect 14nucleotide (nt), 15-nt and >15-nt segments of the RNA, respectively [36]. Importantly, because the very 3 ' end of the RNA is extruded to a narrow pore from the active center of the enzyme during backtracking, the extruded RNA remains inaccessible to RNases increasing in length as backtracking increases [36]. Thus, paused RNAP in either the pre- and post-translocated states as well as at different backtracked distances were monitored over the entire genome. The unique properties of our RNET-seq approach provided an opportunity to dissect the core mechanisms of different types of pausing in living cells.

\section{Results}

\section{Gre factors reduce pausing in the $5^{\prime}$ UTR genome-wide}

We employed RNET-seq on the wild-type (WT) E. coli strain and an isogenic strain deficient in genes for GreA and GreB $(\triangle g r e A B)$. Gre factors and their eukaryotic analog TFIIS rescue backtracked complexes of RNAP [1]. Briefly, the cells were rapidly lysed via spheroplasting, and the transcribing RNAPs were released from the genomic DNA by digestion with DNase I (Fig. 1A). Any ribosomes involved in co-transcriptional translation were separated from RNAP by digestion with RNase A. During the cell lysis heparin was present to inhibit nonspecific binding of RNAPs to DNA and RNA [37]. All RNAPs, including those associated with the fragmented double-stranded DNAs and their $5^{\prime}$-truncated nascent RNAs, were immobilized on $\mathrm{Ni}^{2+}$-NTA beads through the hexa-histidine-tagged $\beta$ ' subunit [38] and then extensively washed with a high-salt buffer (see "Materials and methods"). The purification was done in the native conditions not involving DNA-protein crosslinking. The 5' ends of the transcripts in ECs were trimmed with RNase T1/V1 (V1 digests double-stranded RNAs in nascent transcripts, which are resistant to T1) to leave a minimal length of RNA protected by RNAP (Fig. 1A). The RNases were subsequently removed by further washing of the beads. Next, elution with imidazole generated ECs carrying 6- to 30-nt long transcripts (Fig. 1B; Fig. S1A in Additional file 1). The predominant RNA length distribution was consistent with nucleotide lengths of nascent RNA protected by RNAP from in vitro digestion by different RNases in active and backtracked ECs (Fig. 1B; $\geq 14$ nt) [36]. The imidazole eluate also contained shorter $<14$-nt 

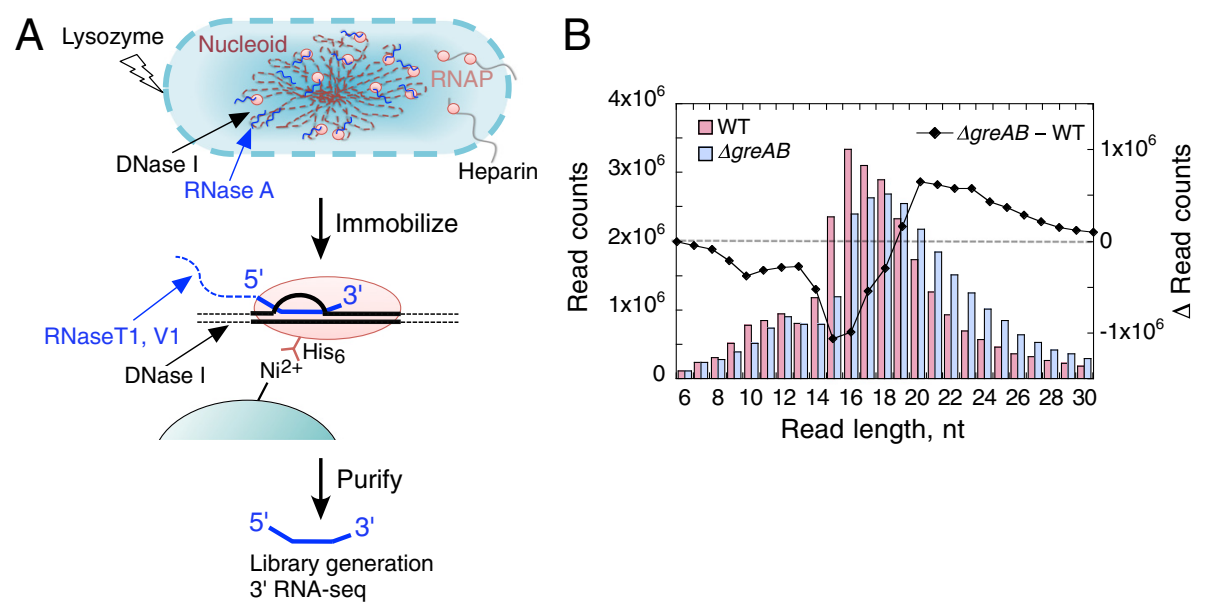

Fig. 1 RNET-seq, the read-length-specific NET-seq approach for the analysis of transcriptional pausing and errors in vivo. (a) An overview of the preparation of the RNA samples for RNET-seq. The 3' RNA transcripts protected by RNAP from RNases were isolated from E. coli cells. (b) Different distribution of read lengths between the WT and $\triangle g r e A B$ cells

RNA species (Fig. 1B; Fig. S1A in Additional file 1), which preferentially mapped to the transcription start site regions of the E. coli genome (Fig. S2 in Additional file 1), indicating that these short reads derived from active transcription initiation complexes and/or moribund abortive initiation complexes [39]. The nascent RNAs isolated from the $\triangle g r e A B$ strain were longer than those from the WT strain (Fig. 1B) and peaked at $18 \mathrm{nt}$ versus $16 \mathrm{nt}$, suggesting an enrichment of backtracked ECs, which is expected to occur in the absence of Gre-dependent 3' RNA cleavage.

We investigated the genome-wide landscape of RNAP pausing by using high quality $\geq 21$-nt sequencing reads (Fig. S3 in Additional file 1) for a subset of the $5^{\prime}$ trimmed nascent transcripts isolated from WT and $\triangle$ gre $A B$ cells. Using the $\geq 21$-nt reads allowed an unambiguous mapping of these reads to the $E$. coli genome compared with their shorter counterparts (see "Materials and methods"). We detected pausing patterns in E. coli genes that are consistent with a previous ChIP-chip analysis [40]; for instance, both methods detected increased RNAP pausing in the promoter-proximal region of the serS gene for seryl-tRNA synthetase (Fig. 2A) [40]. Approximately $50 \%$ of all pause sites in WT and $\triangle$ gre $A B$ cells mapped to mRNA genes versus the rRNA and tRNA genes (Fig. 2B). This number is in sharp contrast to an RNA-seq analysis using total cellular RNA, where $<2 \%$ of the reads in E. coli mapped to mRNA genes while the rest mapped to rRNA and tRNA genes [41]. The dramatic depletion of the rRNA and tRNA transcripts in our RNET-seq data argues that mRNA transcription is much more susceptible to pausing than rRNA and tRNA transcription. This is consistent with the findings that (i) increased density of transcribing RNAPs leads to suppression of backtracking of the leading RNAP by the trailing one [42] and (ii) Nus protein complexes are required for transcription of operons containing rRNA and tRNA genes since these complexes inhibit pausing $[43,44]$.

It has been shown that Gre factors affect transcription initiation, elongation and fidelity [25, 31, 45-48]. However, a role for Gre factors in the global control of transcription pausing in vivo remains poorly understood. We addressed the impact of Gre proteins on pausing using RNET-seq analyses, comparing the nascent transcript levels in the WT and $\triangle$ gre $A B$ cells for each mRNA gene by calculating the number of normalized reads per gene (Fig. S4A in Additional file 1). The value is affected by three parameters: (i) promoter strength, (ii) frequency of pausing, and (iii) duration of pausing during elongation [35]. Note that because the nascent transcript levels were calculated for $\geq 21$-nt reads, paused polymerases within the initiation region (corresponding to <14-nt reads) were not included. We observed a weaker correlation between strains with and without Gre for mRNA reads $(r=0.75)$ compared with rRNA $(r=0.99)$ or tRNA reads $(r=0.89)$ (Fig. S4 in Additional file 1).

To determine if transcription of specific segments of mRNA genes and operons is targeted by Gre factors, we calculated the number of normalized reads for all mRNA genes by dividing them into four separate regions: 50 bases immediately upstream of the start codon (Up); 50 bases just downstream of the start codon (Head); 50 bases just upstream of the stop codon (Tail); and 50 bases just downstream of the stop codon (Down) (Fig. 2C). There was only one significant difference with and without Gre present, and it was found in the Up region of monocistronic operons as well as in the first genes in polycistronic operons (Fig. 2C). Because the median length of 5' UTRs in $E$. coli is 36 bases [49], we suggest that Gre factors 


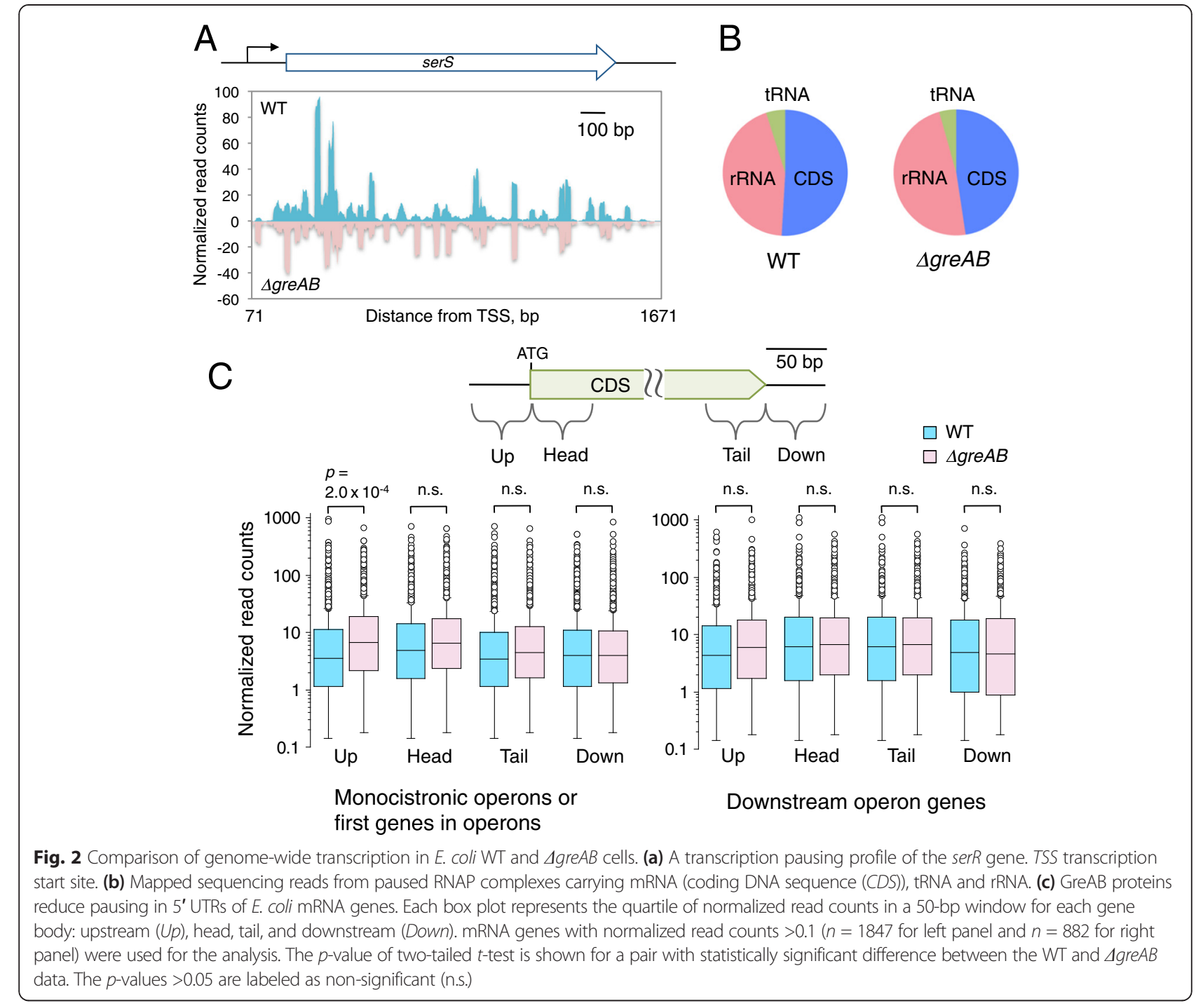

B

suppress RNAP backtrack pausing in the 5' UTRs without having a significant effect on the distal parts of genes and operons. Although we arbitrarily selected 50 bases for the Up region regardless of any variability in the $5^{\prime}$ UTR lengths, we observed stronger suppression of pausing by Gre proteins in Up regions that contained $5^{\prime}$ UTRs with lengths of around 50 bases (Fig. S4B in Additional file 1). This finding is consistent with the proposed role of cotranscriptional translation in suppression of backtrack pauses in E. coli [50].

\section{RNET-seq using $\geq 21$-nt reads identifies robust pausing signals and their non-random distribution}

We examined every base pair position throughout the genome in both orientations as a potential pause site by determining the number (or depth) of reads $(\delta)$ for each mapped genomic position as well as the fraction $\phi$ ) of those reads in which that position is at the 3' RNA end.
To identify robust pause sites by RNET-seq, we selected only those genomic positions where RNAP paused with a frequency $\phi$ that is arbitrarily high $(\geq 0.9$; Fig. S5 in Additional file 1). Thus, $P(\phi, \delta)$, where $\phi$ is the minimal fraction of having 3' RNA ends in the mapped reads and $\delta$ is the minimal read depth for any genomic position. We chose $\delta$ to be 100 for WT and 160 for $\triangle$ gre $A B$, which normalized these respective numbers for each strain since there were 1.6-fold more total reads in the $\triangle \operatorname{gre} A B$ strain. The high $\phi$ parameter allowed us to define a reliable pause-inducing element (PIE) for WT or $\triangle$ gre $A B$ cells (Fig. $3 \mathrm{~A}$ ). The PIEs identified in both strains were different from each other and had high scores for information content, which were similar to other already-known transcription factor motifs in E. coli (Fig. 3A; Tables S1 and S2 in Additional file 2; Method S1 in Additional file 3 for calculation) [51-53]. 


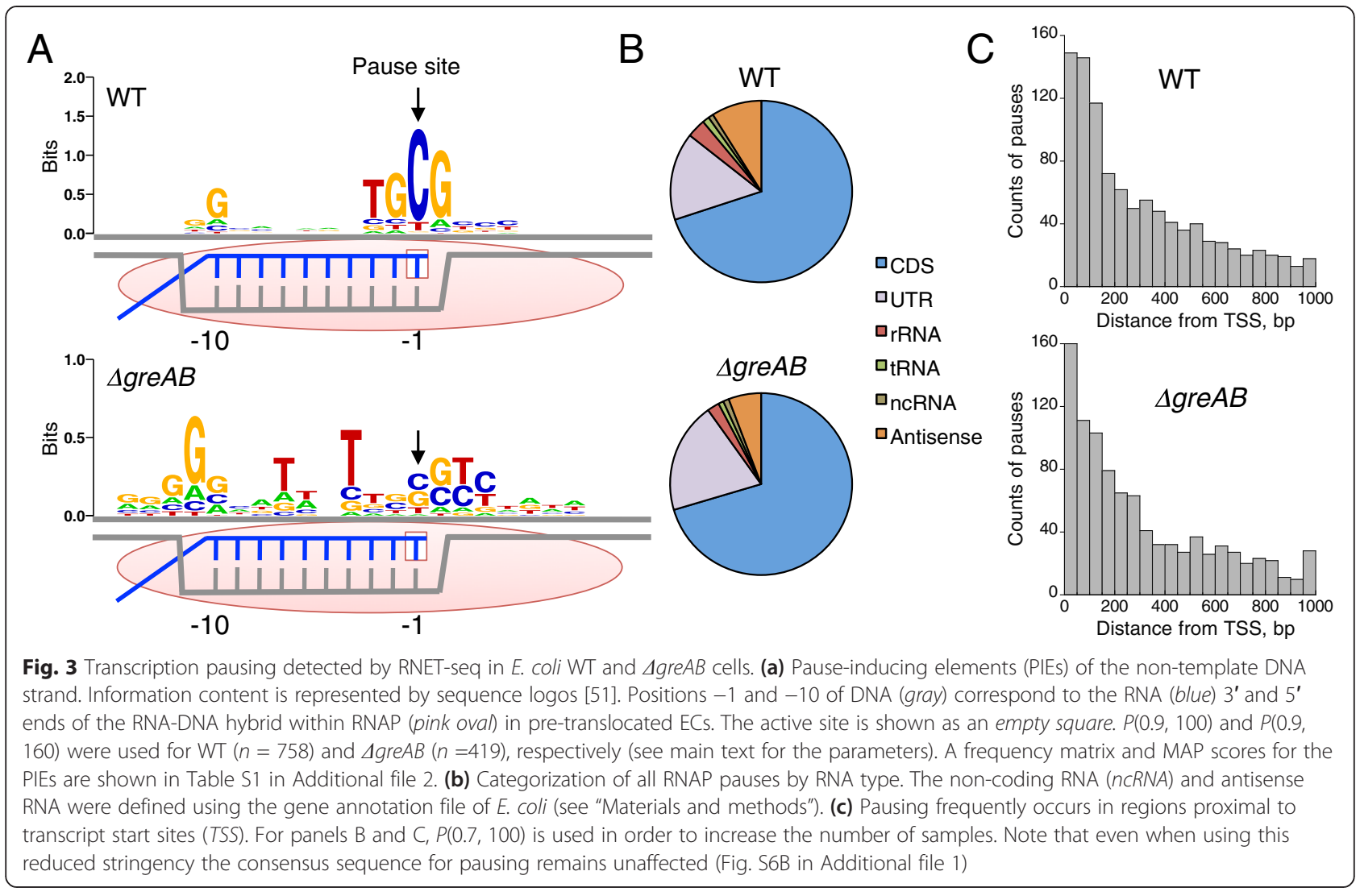

The PIE for the WT strain consisted of the upstream and downstream subelements: (i) a $G_{-10}$ located at the upstream edge of the 10-bp RNA-DNA hybrid in a pretranslocated EC [54]; and (ii) a $\mathrm{TGC}_{-1} \mathrm{G}_{+1}$ sequence spanning the RNAP active center, where the -1 DNA base of the non-template strand corresponds to the 3' RNA base in a paused EC. Notably, the $\mathrm{C}_{-1} \mathrm{G}_{+1}$ sequence accounts for about half of the total score for information content in the motif (Table S1 in Additional file 2). The PIE derived from the $\triangle$ gre $A B$ data consisted of $\mathrm{G}_{-11}$ and $\mathrm{T}_{-4} \mathrm{~T}_{-7}$ (Fig. 3A). Excluding from the $\triangle$ gre $A B$ data those pause sites shared by WT and $\triangle g r e A B$ cells did not significantly change the $\triangle$ gre $A B$ PIE (Fig. S6A in Additional file 1). These data argue that major pause sites found in the WT and the $\triangle$ greAB cells are very different in vivo, contrary to an $E$. coli NET-seq study that made a similar comparison [55]. Our previous studies showed that the weak U-dA base pairs in the RNA-DNA hybrid in bacterial and eukaryotic ECs stimulate RNAP backtracking in vitro $[25,56]$. The similarity between in vitro and in vivo data indicates that lack of Gre proteins in the cell enriched the backtracked RNAP. This enrichment predominantly occurs at the T-rich signal $\left(\mathrm{T}_{-4} \mathrm{~T}_{-7}\right.$ motif) coding for the unstable U-dA base pairs in the EC [12]. The two distinct PIEs observed for WT and $\triangle$ greAB cells were also found when using less stringent $\phi$ and $\delta$ values to define pausing (Fig. S6B in Additional file 1). $95 \%$ (WT) and $94 \%(\triangle g r e A B)$ of the pause sites identified using these parameters showed high uniqueness for the read mapping (Fig. S7 in Additional file 1) [57]. Other pause sites with lower mapping uniqueness were mainly located in the multi-copy rRNA genes or in the multicopy insertion sequence (IS) gene coding for transposase (Fig. S7 in Additional file 1; see "Materials and methods").

Among all types of genes, pausing was primarily detected in mRNA genes in both WT and $\triangle$ gre $A B$ cells (Fig. 3B). Considering that the UTRs are $\sim 10-20$ times shorter than the coding DNA sequences (CDSs) in $E$. coli mRNA, pausing density in the UTRs was found to be higher than in the CDSs. Figure $2 \mathrm{C}$ shows an increased frequency or duration of pausing in $5^{\prime}$ UTRs (50 bp) in $\triangle \operatorname{gre} A B$ compared with WT cells, and Fig. 3C shows no significant difference in the frequencies of those pauses within the $200 \mathrm{bp}$ downstream of transcription start sites. Thus, we suggest that the duration of those pauses was increased due to RNAP backtracking in $5^{\prime}$ UTRs of $\triangle$ gre $A B$ cells. This notion is supported by the $\triangle$ gre $A B$ PIE specifically having the backtracking $\mathrm{T}_{-4}$ $\mathrm{T}_{-7}$ signal coding for a weak RNA-DNA hybrid (Fig. 3A; Fig. S6A in Additional file 1) [12]. 
Putative role of antisense transcription in gene repression Antisense or convergent transcription has been found in all kingdoms of life [58-61]; however, its physiological role remains obscure. Our RNET-seq analysis revealed that polymerase often transcribed in both directions through the same regions. In some cases, two convergent genes were involved, and in others, it appeared that antisense transcription occurred in an annotated noncoding sequence. Pausing during antisense transcription was the third most common type out of all pausing events in E. coli (Fig. 3B). A scatter plot of the normalized read counts obtained in the sense and antisense directions for the 50-bp Tail region showed a slightly negative correlation between antisense and sense transcription (Fig. 4A; the ratios of antisense transcript levels to sense levels were plotted largely across $y=x$, representing positive correlation). This pattern suggests that converging RNAPs interfered with each other. A similar trend was observed in the 50-bp Up and Head regions in genes (Fig. S8A in Additional file 1), indicating a robust interference effect wherever convergent transcription occurred. For example, the $r f a H$ and tatD genes, which are located in a head-to-head orientation on the chromosome, show the same pausing pattern (Fig. 4B). These two genes are expressed at low levels in exponentially growing cells under the conditions we employed for RNET-seq [62, 63]. Notably, $r f a H$ showed a strong cluster of pauses near the $5^{\prime}$ end of the gene (sites 1 and 2 ), which coincided with strong pauses in the antisense direction for the tatD gene (sites 5 and 6). A similar cluster of pauses was observed in tatD where progression of RNAP appeared to be confined to the $5^{\prime}$ part of the tatD gene (sites 3 and 4), which we interpreted as a sign of collision between the sense transcription and the antisense traffic streaming from $r f a H$ (sites 7 and 8).
Interestingly, $\mathrm{RfaH}$ protein is an anti-pausing transcription factor predominantly expressed in stationary growth phase [3]. This implies a potential effect of antisense pausing on the suppression of gene expression under control of the $\mathrm{RfaH}$ protein in the early growth phase. We noted that all pause sites for convergent transcription in $\mathrm{rfaH} /$ tatD were similar to other $\mathrm{G}_{-10 \ldots} \mathrm{C}_{-1} \mathrm{G}_{+1}$ PIEs where unidirectional transcription occurred (Fig. 4B). A similar antisense pattern was observed in the ins $B$ gene coding for the transposase of the IS1 mobile element (Fig. S8B in Additional file 1) [64]. These pauses at IS1 in WT cells represented $\sim 2 \%$ of all pauses identified with parameter values $P(0.9,100)$. We propose that the antisense pausing in IS1 may be essential to prevent spontaneous bursts of transposase production that destabilize $E$. coli genome.

\section{The G-dC base pair at the $5^{\prime}$ end of the RNA-DNA hybrid interferes with translocation}

E. coli RNAP strongly protects the 14-nt segment of the nascent transcript from degradation by RNase $\mathrm{T} 1$ and RNase A in an active post-translocated EC in vitro [36]. Likewise, in the pre-translocated state, RNAP protects the 15-nt transcript, whereas backtracking increases the protection to $\geq 16 \mathrm{nt}$ depending on the backtracking distance [36]. Therefore, each of these different length reads in the RNET-seq contains information about translocation and backtracking states of RNAPs associated with them during elongation and pausing in vivo. We employed this useful information to analyze the dynamics of RNAP translocation and backtracking associated with pausing in vivo.

We compared the individual PIEs for each separate length of RNA read from 14 upto 23 nt across the entire E. coli genome. This single-length RNET-seq analysis

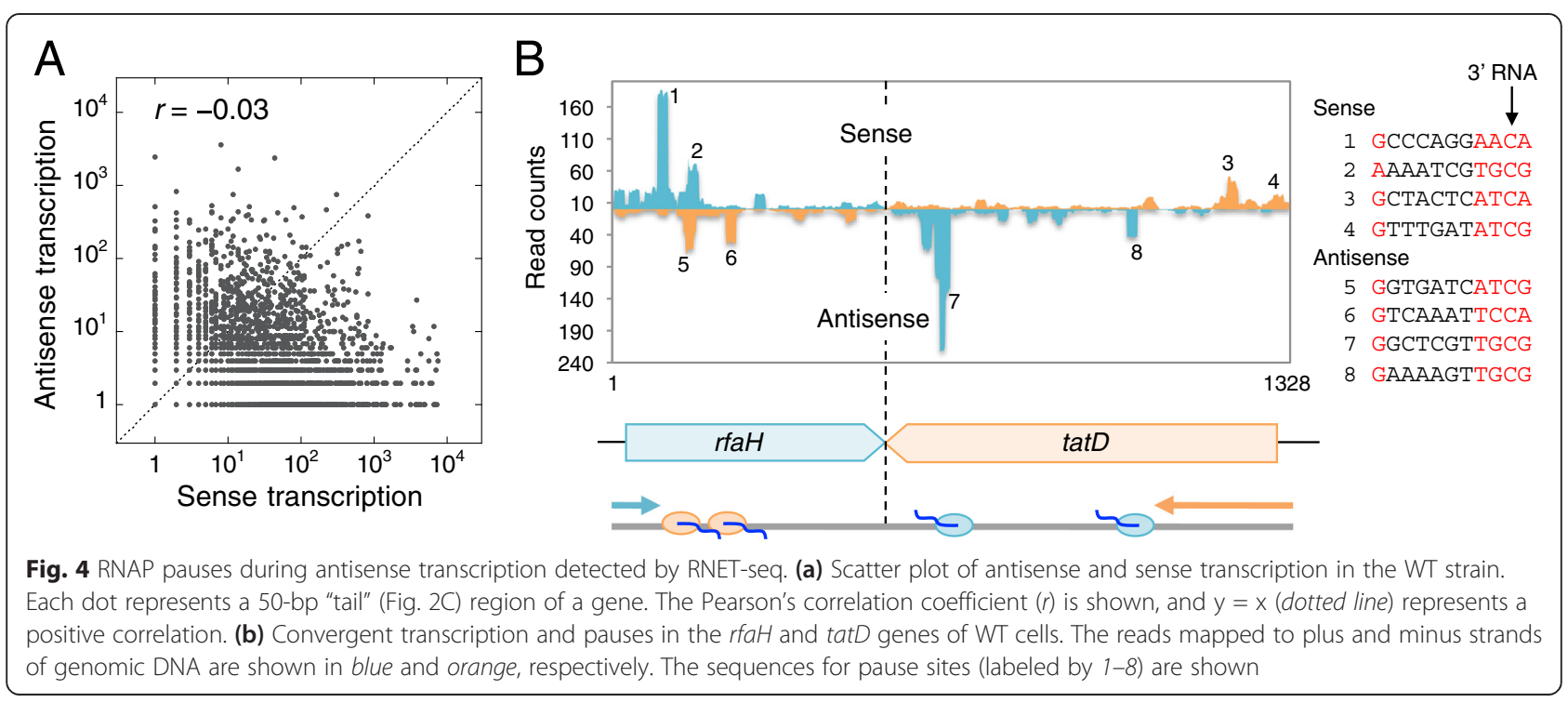


revealed informative differences in distance between the upstream and downstream subelements of the PIEs, which were dependent on the specific read length and corresponding translocation state of RNAP. The observed differences also depended on the presence or absence of Gre factors (Fig. 5A; Figs. S9 and S10 in Additional file 1). In the post-translocated pauses containing 14-nt long reads in WT cells, the $G_{-10}$ signal of the upstream PIE identified using bulk reads $\geq 21$ nt in length was significantly reduced and $\mathrm{G}_{-9}$ was moderately favored (compare Figs. 3A and $5 \mathrm{~A}) . \mathrm{G}_{-9}$ was more prominent in positions with low mapping quality (Fig. S11 in Additional file 1), which we did not investigate further. In WT cells, $G_{-10}$ was the most reliable upstream PIE signal in the pre-translocated state (15-nt long reads) or the 1-bp backtracked state (16-nt long reads) (Fig. 5A). A similar trend was observed in $\triangle g r e A B$

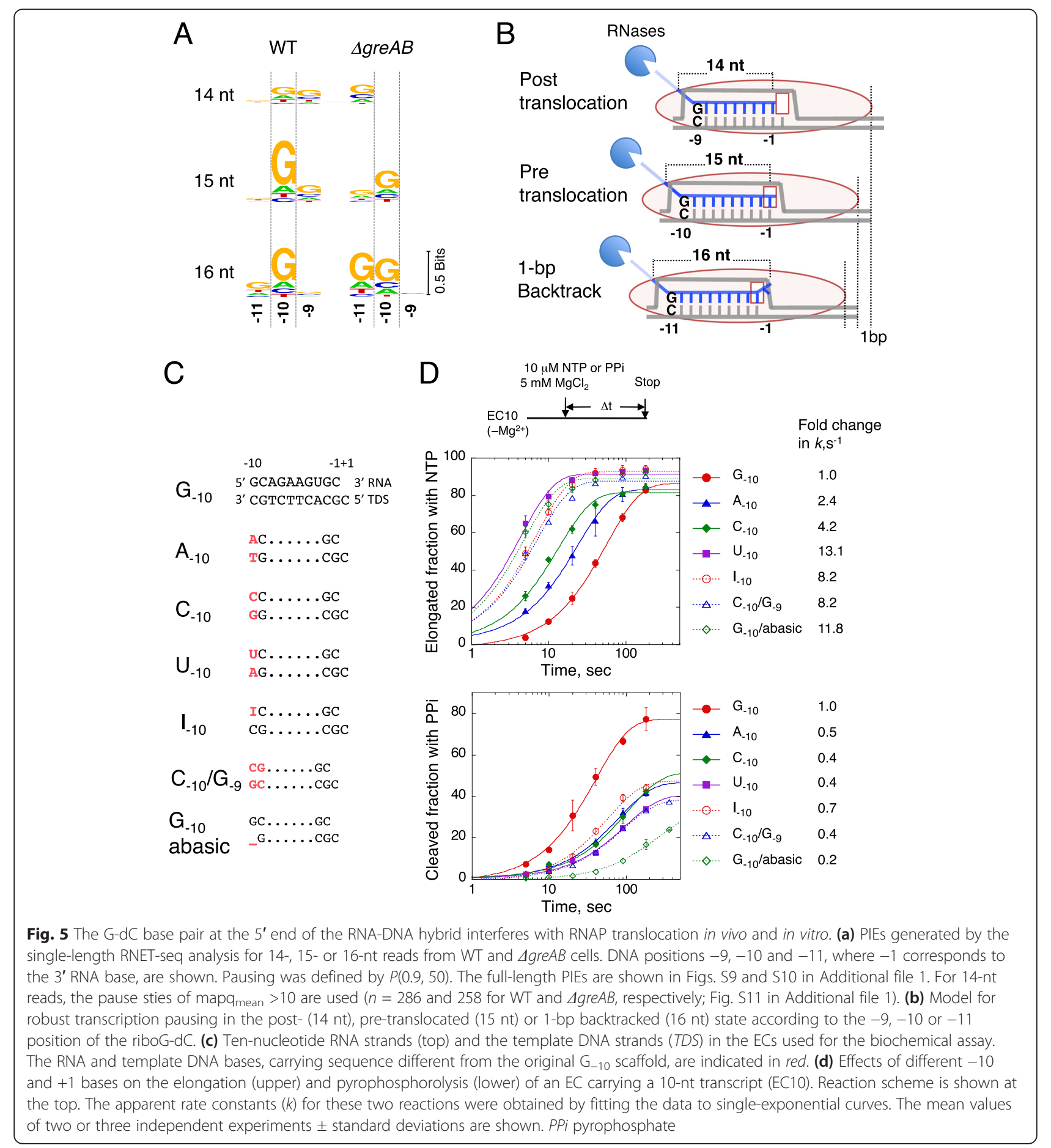


cells except that $G_{-11}$ was the most favored for the $\geq 1$-bp backtracked states (Fig. 5A; Fig. S10 in Additional file 1).

The post-translocated EC of bacterial RNAP has been shown to contain a 9-bp RNA-DNA hybrid [54], which also implies that the pre-translocated and 1-bp backtracked ECs contain a 10-bp hybrid. In the 1-bp backtracked EC, the $3^{\prime}$ nucleotide in the RNA was extruded into the secondary channel in RNAP. Thus, the riboG$\mathrm{dC}$ base pair in the $-9,-10$ or -11 position relative to the 3' RNA end corresponds to the upstream end of the RNA-DNA hybrid in post-translocated, pre-translocated and 1-bp backtracked states, respectively (Fig. 5B). This riboG-dC base pair at the upstream end of the hybrid appears to be very important for pausing to occur in any one of the three translocation registers of RNAP (Fig. 5B). We interpreted that this base pair prevents rapid exchange between any of these states. The favored $\mathrm{G}_{-10}$ in the WT PIE also suggests that Gre-mediated RNA cleavage rapidly rescues backtracked ECs and allows RNA to be elongated back to the pre-translocated pause in WT cells (see "Discussion" for the detailed mechanism).

To validate a role for a riboG- $\mathrm{dC}$ base pair at the upstream end of the RNA-DNA hybrid in pausing, we performed in vitro assembly of ECs with RNAP purified from the $\triangle g r e A B$ strain and synthetic DNA-RNA hybrid scaffolds carrying different bases at the -9 and -10 positions relative to the downstream PIE $\left(\mathrm{TGC}_{-1} \mathrm{G}_{+1}\right.$; Fig. $\left.5 \mathrm{C}\right)$ [25]. The bias in translocation equilibria of these ECs was analyzed by measuring the rates of RNA extension and pyrophosphorolysis [12]. ECs in the pre-translocated state exhibit a characteristic high rate of pyrophosphorolysis and low rate of RNA extension as opposed to their posttranslocated counterparts, which typically exhibit a low rate of pyrophosphorolysis [12]. An EC carrying $\mathrm{G}_{-10}$ showed increased pyrophosphorolysis at $10 \mu \mathrm{M}$ pyrophosphate (PPi), being 1.4-fold more rapid than RNA extension at the same $10 \mu \mathrm{M}$ concentration of NTP (Fig. 5D). In agreement with the in vivo data, this indicates a preferred pre-translocated state for this complex. Substituting $\mathrm{G}_{-10}$ for $\mathrm{A}, \mathrm{C}$, or $\mathrm{U}$ increased the RNA extension and decreased pyrophosphorolysis with $\mathrm{A}_{-10}$ causing less dramatic results than $C_{-10}$ or $U_{-10}$ (Fig. 5D). The dramatic effect of a $G_{-10}$ to $C_{-10}$ substitution on translocation, with only a minor effect on thermodynamic stability of the base pairing, argued against a simple view that the more stable RNA-DNA base pairing at the hybrid end interferes with translocation. A $\mathrm{G}_{-10}$ to I (inosine) ${ }_{-10}$ substitution in the RNA, which altered the riboG-dC hydrogen bond geometry [65], or replacement of $\mathrm{dC}_{-10}$ in template DNA with a non-instructional abasic site (Fig. 5C) also significantly increased the RNA extension rate and reduced pyrophosphorolysis in the complex (Fig. 5D). Thus, we conclude that the particular character of the $5^{\prime}$ riboG-dC base pair of the hybrid was necessary for biasing translocation equilibrium toward the pre-translocated state.

$A \mathrm{G}_{-10} \mathrm{C}_{-9}$ to $\mathrm{C}_{-10} \mathrm{G}_{-9}$ conversion at the upstream end of the hybrid substantially stimulated RNA extension and reduced pyrophosphorolysis in the complex (Fig. 5D). This result indicates that the riboG ${ }_{-9}-\mathrm{dC}$ base pair inclined translocation equilibrium toward the posttranslocated state. Note that the riboG-dC base pair at the $-9,-10$ or -11 position in the post-translocated, pre-translocated, or 1-bp backtracked state, respectively, likely interacts with the same part of RNAP to restrict the EC mobility on DNA in all three transcription registers (Fig. 5B). Taken together, both in vivo and in vitro data argue that interaction of RNAP protein with the riboG-dC base pair at the upstream end of the hybrid interferes with RNAP translocation irrespective of the translocation register of the enzyme. This interference, together with the effect of the CpG downstream element on translocation and/or catalysis, determines RNAP pausing in each translocated state (discussed below).

\section{G-to-A error at the 3' RNA end induces backtrack pausing genome-wide}

It has been reported that a transcriptional G-to-A error in E. coli induces prolonged backtrack pausing of RNAP, which can be the most frequent error in the absence of Gre factors [25]. We used this knowledge to examine if the RNET-seq analysis could detect G-to-A errors enriched in the nascent $3^{\prime}$ transcripts of the paused complexes. Indeed, G-to-A errors were significantly enriched at the 3' RNA ends in $\triangle$ gre $A B$ cells but not in WT cells (Fig. 6A). The G-to-A errors in $\triangle g r e A B$ cells were also dominant among all 12 possible errors types (Fig. 6B). The highest G-to-A error rate $\left(\sim 8 \times 10^{-3}\right)$ was observed at the $3^{\prime}$ ends in the 18-nt reads of $\triangle$ gre $A B$ cells; these errors constituted $\sim 1 \%$ of all pausing events. Importantly, the G-to-A error rate steadily declined as the read length decreased from 18 to $14 \mathrm{nt}$, representing a correlation of the error rate and the backtracking distance (Fig. 6A). This pattern indicates that backtracking was essential for RNAP pausing after the misincorporation. In WT cells, efficient proofreading of the 3' G-to-A errors by Gre factors in the backtracked RNAP appeared to be responsible for their reduction below the RNET-seq detection limit (Fig. 6A). Other types of errors (e.g., C-to-A or C-to-G) were not significantly affected by deletion of Gre factors (Fig. 6B). We interpreted that these errors represent RNET-seq artifacts. Alternatively, these transcription errors could derive from DNA sequences where the 3' RNA-DNA mismatches did not induce RNAP backtracking to prevent their proofreading by Gre factors.

RNET-seq also showed an enrichment of some types of errors at variable distances from the 3' RNA end (Fig. S13 in Additional file 1). For instance, G-to-U errors were 

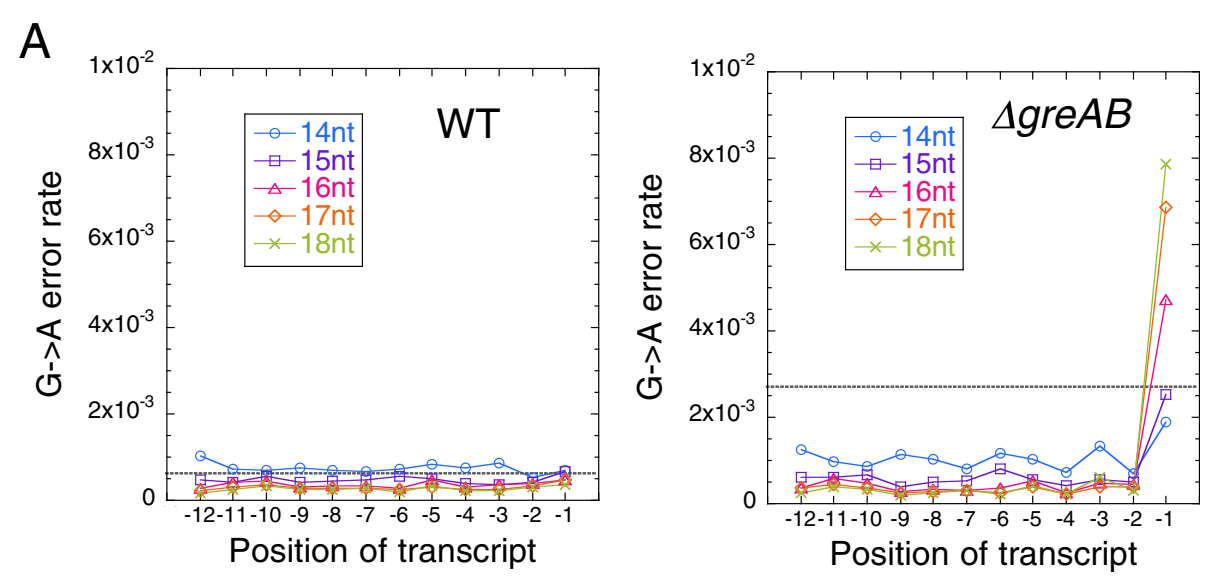

B

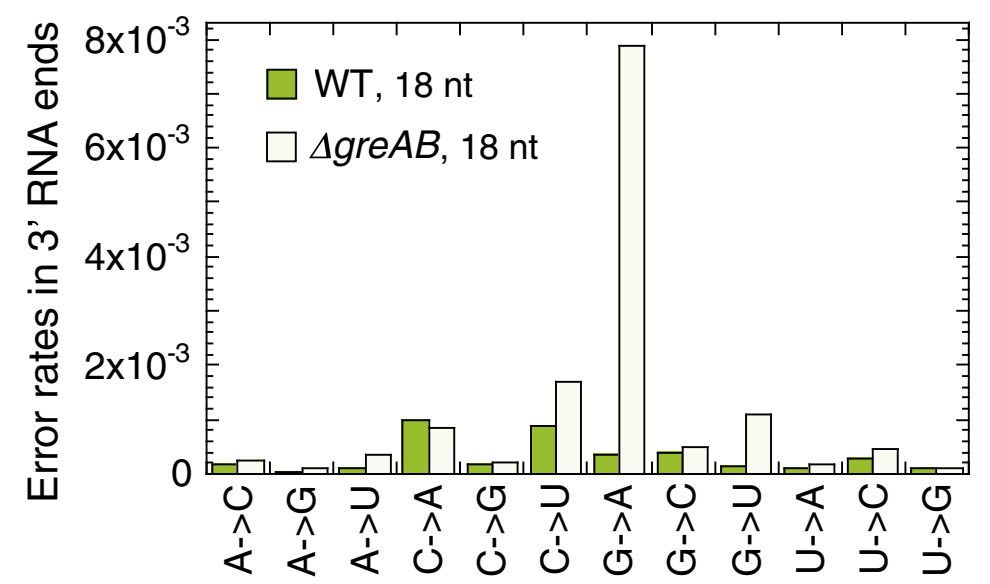

Fig. 6 Transcriptional errors detected by single-length (14-18 nt) RNET-seq. (a) G-to-A error rates at the 3' RNA ends are increased in the absence of Gre factors. Position -1 corresponds to the 3' RNA end. Broken lines represent values for mean error rate + standard deviation in the -12 to -1 positions of the 14- to 18-nt reads. (b) Error rates in the $3^{\prime}$ ends of nascent transcripts detected by single-length (18 nt) RNET-seq

highly enriched between the -8 and -6 positions, with the peak position depending on the read length of RNET-seq. G-to-C, A-to-C, U-to-C and U-to-G errors were also enriched in the -3 positions in $\triangle g r e A B$ cells and this enrichment was also dependent on the read length. Note that the average artificial error rates in our sequencing method were on the order of $10^{-4}$ or less and the sequencing data were of high quality (Figs. S3 in Additional file 1) [25]. These unique properties argue that these errors occurred during transcription in vivo. More extensive biochemical analysis is warranted to understand how different types of transcriptional errors located within the RNA-DNA hybrid in an elongating RNAP induce pausing in vivo.

\section{Discussion}

RNET-seq has identified $\mathrm{G}_{-10}$ and $\mathrm{TGC}_{-1} \mathrm{G}_{+1}$ as robust pausing signals in $E$. coli cells that are similar to pausing sites analyzed by biochemical or single-molecule in vitro studies for E. coli RNAP and yeast/human RNAPII [12,
66, 67]. In human RNAPII, a poly $(G)$ rather than a single $\mathrm{G}$ immediately upstream of the RNA-DNA hybrid was shown to induce strong backtrack pausing rescued by TFIIS, the eukaryotic counterpart of Gre factors [66]. Thus, a core sequence-dependent mechanism for RNAP pausing on bare DNA appears to have similar sequence requirements in prokaryotes and eukaryotes.

How does the $\mathrm{C}_{-1} \mathrm{G}_{+1}$ element spanning the active site of RNAP induce robust pausing in vivo? We previously suggested that increased flexibility of the dCMP sugarphosphate backbone of a CpG dinucleotide in the template DNA strand can cause pausing by interfering with proper alignment of the template DNA base with incoming NTP in the post-translocated state [1, 33]. Similar misalignment of the 3' RNA end with the template base may cause pausing in the pre-translocated state $[1,12$, 68]. This dynamic property of CpG in DNA has been identified by a variety of methods [69-71]. A recent deep-sequencing study of transcription in mammalian cells revealed that RNAPII pausing frequently occurred 
in gene bodies containing CpG repeats [72]. Importantly, CpG methylation reduces flexibility of the sugar moiety of the $\mathrm{dC}[73,74]$, and also reduces pausing of E. coli RNAP in vitro [75]. Our in vitro experiment using a 5methyl- $\mathrm{dC}_{+1}$ residue introduced into the pausing motif moderately increased RNA extension rate and strongly reduced efficiency of pyrophosphorolysis (Fig. S12 in Additional file 1). Thus, the 5-methyl- $\mathrm{dC}_{+1}$ in the template DNA of the PIE appeared to shift the translocation equilibrium to the post-translocated (pyrophosphateresistant) state.

Our work shows that G-to-A errors at the 3' RNA ends cause backtrack pausing of RNAP genome-wide (Fig. 6). Interestingly, this in vivo effect was much stronger for G-to-A errors than for any other error types in $E$. coli (Fig. 6B; Fig. S13 in Additional file 1), suggesting a common mechanistic origin of pausing and AMP misincorporation on $\mathrm{C}_{-1} \mathrm{G}_{+1}$ sequence in the non-template strand $\left(\mathrm{G}_{-1} \mathrm{C}_{+1}\right.$ in the template strand). We propose a mechanism that explains a link between the frequent Gto-A errors and pauses in the PIE (Fig. 7A). An encounter with the $\mathrm{G}_{-10 . . .} \mathrm{C}_{-1} \mathrm{G}_{+1}$ sequence induces RNAP pausing. During pausing, incorporation of the next cognate GMP decreases while the AMP misincorporation increases, as has been previously shown in vitro [25]. Both pausing and misincorporation derive from the flexible sugar backbone of the $\mathrm{dC}_{+1}$ template residue (Fig. 8A). The lack of structural constraint interferes with the canonical alignment of GTP with dCMP in the active center and makes the non-canonical alignment of ATP with dCMP more tolerated. Alternatively, the increased flexibility of the dCMP may induce its temporary withdrawal from the active site to stimulate the AMP misincorporation according to the A-rule synthesis recently reported for multi-subunit RNAPs during transcription on abasic DNA sites [76]. The resultant formation of an A-dC mis-pair at the 3' RNA end induces a prolonged backtracked pause [25], allowing its detection by RNETseq in $\triangle$ gre $A B$ cells. Such stable backtrack pauses are major threats to genome stability due to their ability to block DNA replication, which can lead to double strand breaks in DNA (Fig. 7A) [22, 24]. Gre factors stimulate rapid removal of the mis-paired 3' AMP residue, allowing RNAP to resume elongation at the original PIE (G. $\left.{ }_{10 . .} C_{-1} G_{+1}\right)$. We confirmed this model by showing that a $\mathrm{C}$ residue in the 3 '-penultimate RNA residue was favored in the reads containing the G-to-A error at the 3' residue, but not in the error-free reads in the PIE derived from backtracked reads (18 nt) in $\triangle$ gre $A B$ cells (Fig. 7B, C). Thus, our data are fully consistent with the model that both pausing and AMP misincorporation are enhanced at the $\mathrm{C}_{-1} \mathrm{G}_{+1}$ sequence (Fig. 7A).

We showed in vivo and validated in vitro that the G$\mathrm{dC}$ base pair at the upstream end of the RNA-DNA hybrid interferes with the forward translocation of RNAP (Fig. 5). An X-ray structure of bacterial EC identified lid
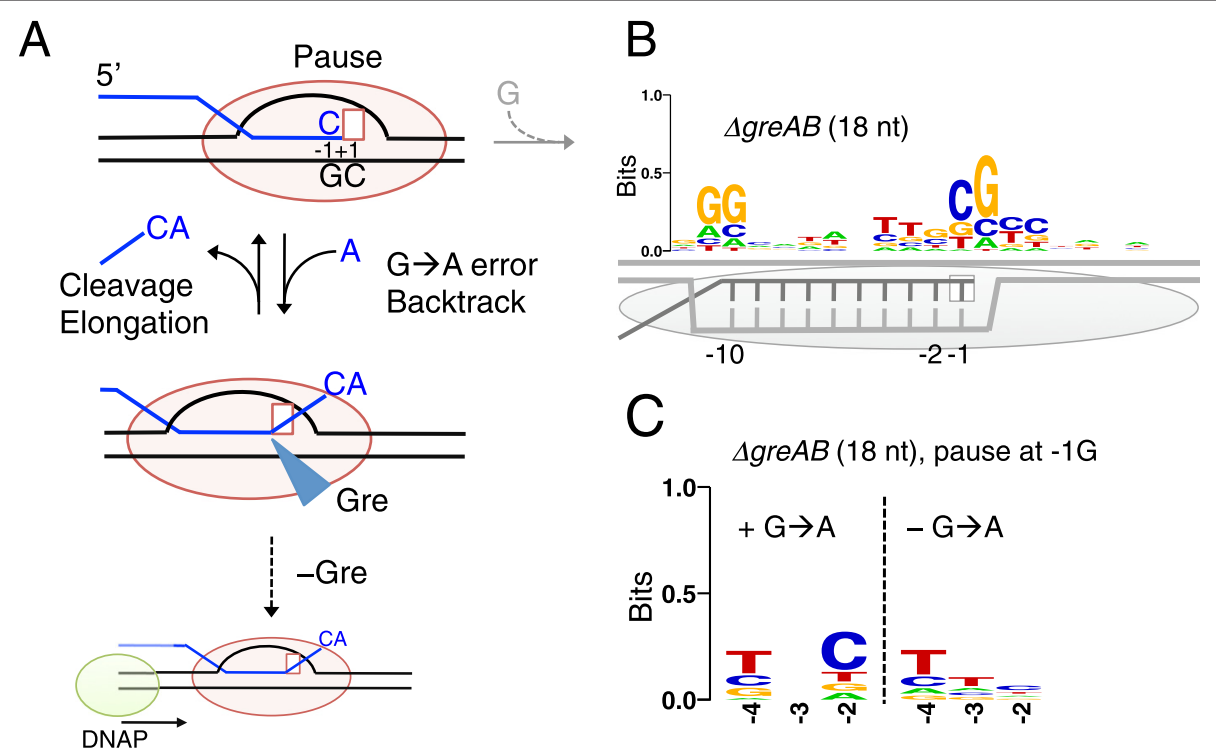

Fig. 7 Transcriptional pauses and errors frequently occur at CpG sequences in vivo. (a) G-to-A error at the 3' RNA end induces backtracking of RNAP in the $\mathrm{C}_{-1} \mathrm{G}_{+1}$ motif. These pauses are rescued and the errors are corrected by Gre factors. In the absence of Gre factors, the backtracked RNAP imposes a strong barrier to a replicating DNA polymerase (DNAP) leading to double-strand DNA breaks [22, 24]. (b) The PIE of the non-template DNA in the $\triangle$ greAB, which is composed of 1,555 pause sites identified by parameters $P(0.9,50)$. The pre-translocated RNAP (gray oval) and the RNA-DNA hybrid are shown. (c) The $3^{\prime}$-penultimate $C$ residue in the nascent RNA is favored when a G-to-A error(s) occurs at the 3' G residue in $\triangle$ greAB cells. The two groups for the PIE are shown: the left-side group represents reads containing $\geq 1$ G-to-A errors at the $G_{-1}(n=127)$ and the right-side group represents the reads containing no error at the $\mathrm{G}_{-1}(n=162)$. For panels $\mathrm{B}$ and $\mathrm{C}, 18 \mathrm{nt}$ reads were used 


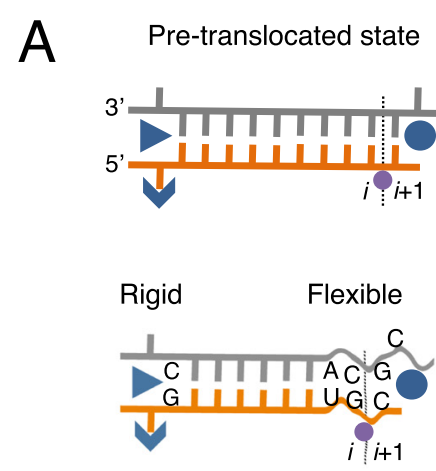

Post-translocated state
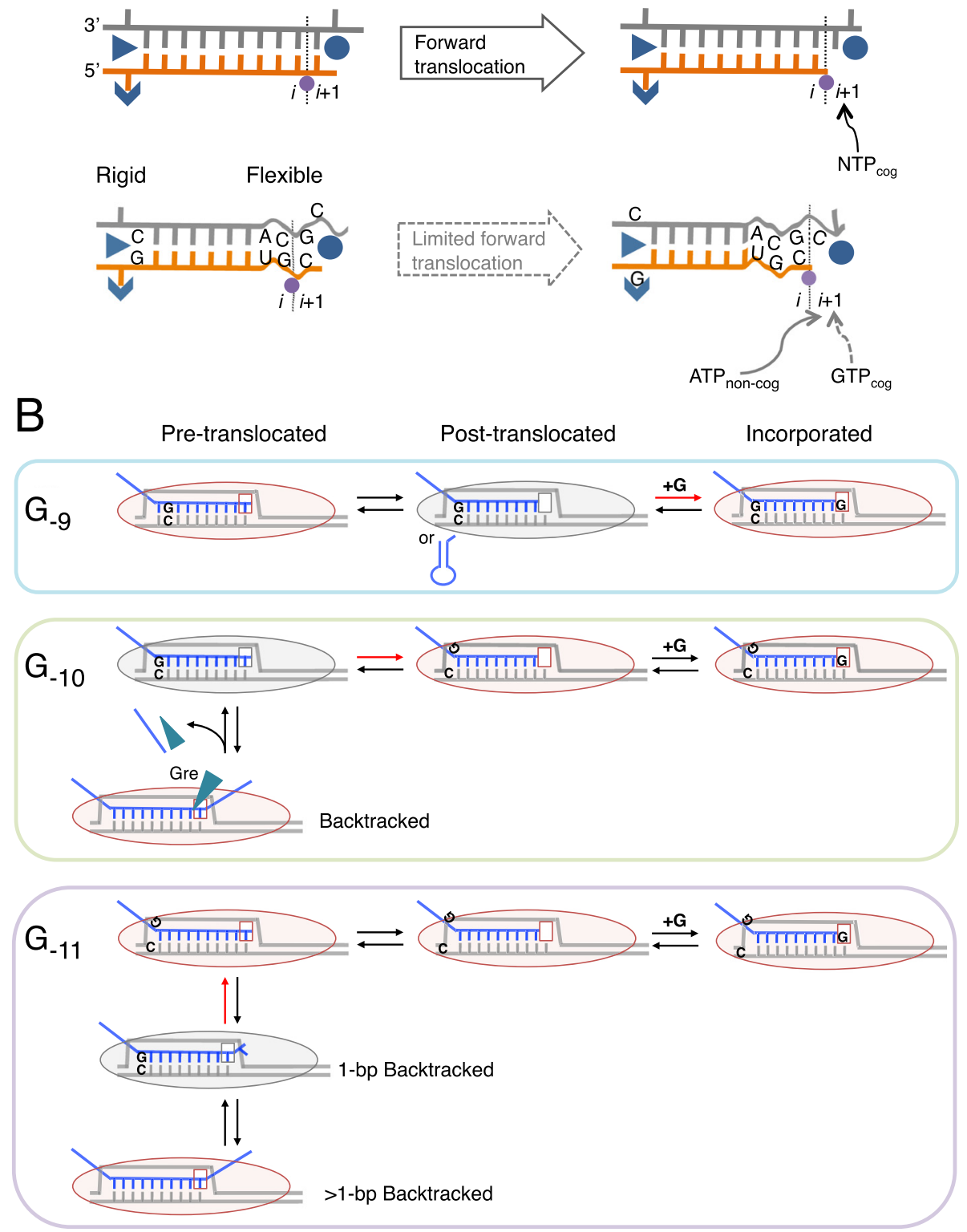

Fig. 8 Structural and kinetic models of transcription pausing in vivo. (a) Structural model. RNAP elongation in a pause-free sequence (top) or the PIE (bottom) is shown. RNA (orange), template DNA strand (gray), catalytic $\mathrm{Mg}^{2+}$ (magenta), and two RNAP domains (blue) involved in the 5' RNA separation from the RNA-DNA hybrid, i.e., Switch 3 (arrow head), lid (triangle) domain, and the bridge helix of RNAP (blue circle) are shown. The 3' RNA-binding site $(i)$ and the NTP binding site $(i+1)$ are also indicated. The $3^{\prime}$ ACGC $5^{\prime}$ sequence in the template DNA and the complementary 5' UGC 3' RNA sequence increase the flexibility of their backbones, which decreases cognate GTP (GTP cog) addition and increases non-cognate ATP (ATP non-cog) addition to the $3^{\prime}$ RNA end. The two RNAP domains can interact with riboG-dC at the upstream end of the hybrid, which interferes with the hybrid movement through the catalytic cleft of RNAP. (b) Kinetic model. RNAP pauses in the post-translocated (G-9 or RNA hairpin, top), pre-translocated $\left(\mathrm{G}_{-10}\right.$, middle), and backtracked states $\left(\mathrm{G}_{-11}\right.$, bottom). RNAPs with the $i+1$ NTP binding site are shown (oval shapes with empty squares). Gre factors are indicated by cyan triangles. Post- and pre-translocated pauses were mainly observed in WT cells, and backtracked pauses were observed in $\triangle$ greAB cells. The rate-determining steps during elongation are indicated by red arrows. The RNAP conformations captured by RNET-seq are indicated by gray ovals. Note that the GreAB-dependent cleavage, which occurs between $i$ and $i+1$ sites, ultimately converts the backtracked state to the post-translocated state. This state is rapidly converted back to the pre-translocated state prior to the next NTP binding and bond formation at the $i+1$ site. The presence of activation energy much higher than $k_{\mathrm{B}} T$ in each rate-determining step is assumed for the kinetic description of pausing in vivo [1, 39] 
and Switch-3 domains in RNAP interacting with RNA nucleotides at the upstream edge of the RNA-DNA hybrid (Fig. 8A) [54]. A biochemical study showed that amino acid changes in these domains affected RNAP catalytic activity and translocation [77]. More work is needed to determine the impact of the G-dC base pair at the end of the hybrid on translocation blocking at the PIE. This block may derive from a specific interaction of the $\mathrm{G}-\mathrm{dC}$ base pair with the RNAP domain(s).

The principal finding of this work is that the location of the $\mathrm{G}_{-10}$ nucleotide relative to the $\mathrm{TGC}_{-1} \mathrm{G}_{+1}$ motif in the PIE fluctuates over a 3-bp distance and these fluctuations correlate with the length of the RNET-seq reads (Fig. 5). Because the read length depends on the translocation state of RNAP during pausing in vivo, this finding served as a foundation for a kinetic model for the robust pausing mechanism in E. coli (Fig. 8B). In paused RNAP, riboG residue at $-9,-10$, or -11 nt position $\left(G_{-9}\right.$, $G_{-10}$, or $G_{-11}$ ) is located at the same distance from RNAP active center in the post-translocated, pretranslocated, or 1-bp backtracked state, respectively (Fig. 8B). The $G_{-9}, G_{-10}$, or $G_{-11}$ that pairs with $d C$ in the template at the upstream end of the RNA-DNA hybrid determines the rate of escape from each of these states during transcription. The $\mathrm{G}_{-9}$ stabilizes the posttranslocated state before backtracking (Fig. 8B). The effect of $\mathrm{G}_{-9}$ on pausing is minor compared with that of the $\mathrm{TGC}_{-1} \mathrm{G}_{+1}$ motif, which causes a misalignment of GTP with the template $\mathrm{dC}$ residue leading to pausing (Fig. 8A). The $\mathrm{G}_{-10}$ strongly interferes with forward translocation but allows backtracking followed by rapid cleavage of the extruded 3' RNA in the presence of Gre factors (Fig. 8B). The rapid cleavage and the slow translocation prevent the escape of RNAP from the pretranslocated state even in the presence of Gre factors. The $G_{-11}$ at the upstream end of the RNA-DNA hybrid is important to stabilize backtracked states. Note that $G$ ${ }_{-11}$ in a 1-bp backtracked complex corresponds to $G_{-10}$ in the pre-translocated complex (Fig. 5B). As $G_{-10}$ inhibits forward translocation, $G_{-11}$ inhibits conversion of the 1-bp backtracked state to the pre-translocated state, thereby favoring continued backtracking in the upstream direction. In WT cells, Gre proteins rapidly cleave the RNA in the backtracked state, allowing RNAP to enter back into the pre-translocated state with $G_{-10}$ present. In contrast, lack of the RNA cleavage in $\triangle$ gre $A B$ cells leads to a predominantly backtracked EC pool with $G_{-11}$ present. Taken together, a bipartite pausing sequence, $G_{-9}$, $\mathrm{G}_{-10}$, or $\mathrm{G}_{-11}$ with $\mathrm{TGC}_{-1} \mathrm{G}_{+1}$ can confer pauses in all translocated states of RNAP in vivo.

In $\triangle g r e A B$ cells, the weak U-dA base pairs in the $-4 /-7$ positions of the hybrid contribute to further backtracking by thermodynamically destabilizing the non-backtracked complexes [78] or via a specific conformation of the hybrid with the $-4 /-7$ U-dA base pairs that kinetically favors backtracking (Fig. 3A). Indeed, the $\mathrm{T}_{-4} \mathrm{~T}_{-7}$ but not $\mathrm{G}$ ${ }_{-11} \ldots \mathrm{C}_{-1} \mathrm{G}_{+1}$ element of the PIE was reduced in the weak backtrack pausing signals (compare the $\triangle$ gre $A B$ PIEs between the high and low $\phi$ parameter; Fig. S6B in Additional file 1). The observed preference for the U-dA base pairs in the $-4 /-7$ positions of the hybrid seems to disagree with the thermodynamic model for pausing, which suggests that the unstable U-rich RNA-DNA hybrid is merely required for RNAP backtracking [78]. Several well-characterized backtracking signals for bacterial RNAP and eukaryotic RNAPII contain the uniform runs of $U$ residues in the RNA $[20,79,80]$. This discrepancy may indicate that $E$. coli cells have evolved not to contain strong PIEs made of a combination of $T$ tracts followed by the $C$ ${ }_{-1} G_{+1}$ sequence to avoid collisions of stably backtracked RNAPs with replication machineries.

While this work was in progress, Larson et al. [55] reported NET-seq analysis of a consensus pausing sequence in $E$. coli that turned out to be very similar to the PIE identified by us. This group showed an enrichment of RNAP pausing at the translation start sites (i.e., the AT ${ }_{-1} \mathrm{G}_{+1}$ motif) in E. coli genes, which was interpreted as a key mechanism for synchronization of transcription and translation [55]. In contrast to their conclusion, we found only a few $\mathrm{AT}_{-1} \mathrm{G}_{+1}$ sequences compared with major TGC ${ }_{-1} G_{+1}$ sequences in pause sites: seven of all the robust 758 pause sites $(<1 \%)$ that we identified by RNET-seq contained an $\mathrm{AT}_{-1} \mathrm{G}_{+1}$ motif and only two out of these seven $\mathrm{AT}_{-1} \mathrm{G}_{+1}$ pause sites were located at the ATG start codon (lpxD and $p g k$ genes). Larson et al. also showed no contribution of Gre factors to pausing in vivo and argued that the pausing in the pre-translocated state does not accompany backtracking of RNAP [55]. In contrast, our analysis of the link between pausing and the translocation state of RNAP strongly suggests that pre-translocated pausing is typically in a dynamic equilibrium with backtracking and 3' RNA cleavage by Gre factors (Figs. 5 and 8B). The transient pre-translocated pause equilibrated with backtracking guarantees that pausing may affect gene expression even at normal intracellular concentrations of $\mathrm{PPi}$, in which the pre-translocated paused state should have a very short half-life. Indeed, our in vitro measurements revealed that $\sim 100 \mu \mathrm{M}$ PPi completes pyrophosphorolysis of the 3' RNA base within seconds of forming the pretranslocated complex that disfavors backtracking (data not shown). Thus, any static pre-translocated pause will be rapidly reversed by pyrophosphorolysis in vivo, thereby grossly limiting its dynamic range and regulatory impact. Taken together, a dynamic rather than a static model for pre-translocated pauses is necessary to understand the physiologically relevant pausing.

More recently, Vvedenskaya et al. [81] identified a robust $\mathrm{G}_{-10} \mathrm{Y}_{-1} \mathrm{G}_{+1}$ pausing signal in $E$. coli by conventional 
NET-seq. This work employed a mutant $E$. coli strain carrying D446A substitution in the RNAP $\beta$ subunit [81]. They argued that the D446 residue interacts with $G_{+1}$ of the non-template DNA, facilitating forward translocation, which promoted read-through of paused RNAPs. However, we noted in their published data that the $\beta \mathrm{D} 446 \mathrm{~A}$ mutation led to a major increase in both $\mathrm{C}_{-1}$ and $\mathrm{G}_{+1}$ of the pausing motif with only a minor effect on their ratio [81]. Thus, the $\beta D 446 A$ RNAP was sensitive to the $C_{-1} G$ ${ }_{+1}$ neighbor rather than to $G_{+1}$ alone, consistent with previous extensive in vitro analyses of pausing signals for $E$. coli RNAP [1, 12, 66, 67]. We note that our RNET-seq analysis, in which we employed only single read lengths, detected some fluctuation in the ratio of $G_{+1}$ to $C_{-1}$ at pause sites depending on the RNA length in the complex. This fluctuation correlated with translocation register and the presence of Gre factors, indicating its relevance to transcription pausing (Figs. S9 and S10 in Additional file 1). This phenomenon indicates the presence of several additional pausing mechanisms related to the $\mathrm{C}_{-1} \mathrm{G}_{+1}$ neighbor, including the proposed interaction of the $G_{+1}$ base in the non-template DNA strand with the D446 residue of the RNAP $\beta$ subunit [81]. More analysis will be needed to elucidate the contributions of $\mathrm{C}_{-1}$ and $\mathrm{G}_{+1}$ to different classes of pausing signals.

\section{Conclusions}

We present several pausing mechanisms governed by a bipartite RNA-DNA hybrid consensus sequence, which consists of an upstream part, a $G$ at either $-9,-10$ or $-11 \mathrm{nt}$ from the $3^{\prime}$ RNA end, and a downstream part, a $\mathrm{C}_{-1} \mathrm{G}_{+1}$ flanking the active center. The upstream $\mathrm{G}$ position determines whether pausing occurs in the post-, pre-translocated or backtracked state, respectively. We suggest that pauses have multiple regulatory roles during transcription of the $5^{\prime}$ UTR regions of genes and during antisense transcription. Using this mechanism, RNAP pausing and its regulation by trans-acting factors can be optimized to suit different genomes with different GC content and $\mathrm{CpG}$ repeats that are broadly present in eukaryotes [82]. Backtracking of RNAP at these sequences imposes a strong roadblock to DNA replication leading to DNA double strand breaks [21]. By rescuing the backtrack pauses, Gre/TFIIS factors would be essential for maintaining genome integrity as suggested previously [1, $25,83,84]$.

We also reveal that DNA sequences for the predominant G-to-A transcription errors in E. coli coincide with the $\mathrm{CpG}$ pausing motif throughout the $E$. coli genome. Thus, the CpG-enriched domain found in chromosomes may play a special role associated with prolonged RNAP backtracking in combination with an elevated error frequency. The CpG domain may compromise genome integrity due to elevated rates of collisions between replication and backtracked transcription complexes via stable R-loop formations [22, 24]. It has been shown that R-loops are increased in unmethylated human CpG islands [85]. Pausing sequences identified for human RNAPII in vitro are similar to the PIE in E. coli [66]. Thus, transcription pausing followed by the G-to-A error may be a source of the R-loops in CpG islands and transcription of these islands may significantly contribute to transcription mutagenesis in both prokaryotes and eukaryotes [86].

\section{Materials and methods \\ Bacterial culture}

E. coli cells carrying the rpoC gene coding for the Cterminal-hexahistidine-tagged $\beta$ ' subunit, NB854 (W3110 rpoC-6xHis::kan gal490) or NB959 (W3110 rpoC-6xHis:: kan greA::tet, greB::amp) were constructed by $\lambda$ Redmediated recombination (see Method S2 in Additional file 3 for detail). To prepare WT and $\triangle$ greA/greB cells for extraction and isolation of RNAP complexes, cells were grown in $\sim 300 \mathrm{ml} \mathrm{LB}$ broth $+25 \mu \mathrm{g} / \mathrm{ml}$ kanamycin with shaking at $37^{\circ} \mathrm{C}$ until an $\mathrm{OD}_{600}$ of $\sim 0.5$. The cells (150 mg wet weight) were harvested by centrifugation at $6,500 \times g$ for 4 minutes at $4{ }^{\circ} \mathrm{C}$, divided into three $1.5 \mathrm{ml}$ tubes, flash frozen in liquid nitrogen, and stored at $-80{ }^{\circ} \mathrm{C}$.

\section{Rapid breakdown of cells and nucleoids}

Each tube of the cell pellet stored at $-80{ }^{\circ} \mathrm{C}$ was resuspended in $650 \mu \mathrm{l}$ TES buffer $(10 \mathrm{mM}$ Tris- $\mathrm{HCl}, \mathrm{pH} 7.5$, $1 \mathrm{mM}$ EDTA, $100 \mathrm{mM} \mathrm{NaCl}, 0.1 \%$ TritonX 100 and 0.2 $\mathrm{mM}$ PMSF) at room temperature. The cell suspension was mixed with $100 \mathrm{kU}$ Ready-Lyse Lysozyme (Epicentre) and $50 \mu \mathrm{g}$ RNase A (Sigma) and incubated for 5 minutes, allowing rapid cell lysis. To digest the genomic DNA, 62.5 U of DNase I (Roche) was added with heparin at a final concentration of $250 \mu \mathrm{g} / \mathrm{ml}$ and $74 \mu \mathrm{l}$ of $10 \mathrm{x}$ DNase I buffer (100 $\mathrm{mM} \mathrm{MnCl}_{2}$ and $100 \mathrm{mM}$ Tris- $\mathrm{HCl}$, $\mathrm{pH}$ 7.5). All were added to the mixture and incubated for 10 minutes at room temperature. To remove cell debris, the mixture was centrifuged at $20,000 \times g$ for 3 minutes at $4{ }^{\circ} \mathrm{C}$ and the supernatant was collected into a new $1.5 \mathrm{ml}$ tube.

\section{Purification of elongation complexes}

The supernatant including ECs were immobilized on a $50 \%$ suspension of $\sim 155 \mu \mathrm{Ni}^{2+}$-NTA agarose (Qiagen) pre-equilibrated with the binding buffer $(0.5 \mathrm{M} \mathrm{NaCl}, 5$ $\mathrm{mM}$ imidazole, $5 \%$ glycerol, $20 \mathrm{mM}$ Tris- $\mathrm{HCl}, \mathrm{pH} 7.9$ and $1 \mathrm{mM} \mathrm{2-mercaptoethanol)} \mathrm{with} \mathrm{shaking} \mathrm{for} 10 \mathrm{mi}$ nutes at $4{ }^{\circ} \mathrm{C}$. The immobilized ECs were washed at $4{ }^{\circ} \mathrm{C}$ five times with the wash buffer $(1 \mathrm{M} \mathrm{NaCl}, 15 \mathrm{mM}$ imidazole, $5 \%$ glycerol, $20 \mathrm{mM}$ Tris- $\mathrm{HCl}$, pH 7.9 and $1 \mathrm{mM}$ 2-mercaptoethanol), and then washed twice with the 
nuclease buffer $(40 \mathrm{mM} \mathrm{KCl}, 15 \mathrm{mM}$ imidazole, $20 \mathrm{mM}$ Tris-HCl, pH 7.9, $0.3 \mathrm{mM} \mathrm{MgCl} 2,5 \%$ glycerol and $1 \mathrm{mM}$ 2-mercaptoethanol). Any DNA unprotected by RNAP was cleaved by the addition of $0.4 \mathrm{U}$ RNase V1 (Invitrogen), 0.7 U RNase T1 (Sigma), and 5 U DNase I (Takara Bio) to the immobilized complexes with incubation for 7 minutes at room temperature. The nuclease-treated complexes were washed twice with the wash buffer, twice with the $\mathrm{MgCl}_{2}-$ free nuclease buffer at $4{ }^{\circ} \mathrm{C}$, and eluted by adjusting the concentration of imidazole to $100 \mathrm{mM}$ in the presence of 30 U SUPERase RNase inhibitor (Ambion) followed by shaking for 10 minutes at $4{ }^{\circ} \mathrm{C}$.

To test the functional RNAP activity of the purified ECs, $5 \mathrm{mM} \mathrm{MgCl}_{2}$ and $0.5 \mathrm{mM} \mathrm{NTP} \pm 8 \mu \mathrm{M}$ GreA and $4 \mu \mathrm{M}$ GreB were added to the complexes (not used for RNET-seq) and incubated for 5 minutes at $37{ }^{\circ} \mathrm{C}$ to follow RNAP transcription. To visualize RNA and DNA species that were associated with the ECs, the samples were heat-denatured for 10 minutes at $70{ }^{\circ} \mathrm{C}$ and either $50 \mathrm{U} / \mathrm{ml}$ DNase I or $5 \mu \mathrm{g} / \mathrm{ml}$ RNase A was added to the sample to digest the DNA or RNA in the sample, respectively. The samples were subjected to PAGE with 15 $\%$ TBE-Urea gels (Invitrogen) followed by staining with SYBR Gold (Invitrogen) according to [87] (Fig. S1B in Additional file 1). ECs carrying 14- to 20-nt long transcripts resumed elongation when incubated in vitro with NTPs and purified GreA and GreB proteins (Fig. S1B in Additional file 1). The fraction of ECs that resumed transcription after treatment with Gre protein appeared to be larger in $\triangle g r e A B$ compared with WT cells (Fig. S1C in Additional file 1). A substantial fraction of the initiation complexes carrying RNAs $\leq 13$ nt required Gre proteins to resume transcription in both WT and $\triangle$ gre $A B$ cells. This fraction may include moribund initiation complexes that require Gre proteins to enter productive elongation $[39,48]$.

\section{RNA extraction}

The $200 \mu$ l eluate from the $\mathrm{Ni}^{2+}$-NTA agarose was mixed with an equal volume of pre-warmed phenol/chloroform/ isoamylalcohol (PCI; 25:24:1) and incubated for 2 minutes at $70{ }^{\circ} \mathrm{C}$. The mixture was centrifuged, and RNA and DNA were precipitated with isopropanol from the supernatant according to [87]. The pellet was dissolved in $30 \mu \mathrm{l}$ DNase I buffer with 5 U DNase I (Takara Bio) and 20 U SUPERase and incubated for 10 minutes at room temperature. RNA was separated from the digested DNA by the PCI extraction and the RNA was precipitated by isopropanol. The pellet was dissolved in diethylpyrocarbonate-treated water and used for cDNA synthesis.

\section{Library construction and sequencing}

cDNA libraries of the nascent RNAs were constructed according to [87]. Briefly, a pre-adenylated DNA linker was ligated onto $\sim 1 \mu \mathrm{g}$ of the purified nascent RNA. The RNA-fragmentation step was skipped in our protocol. Reverse transcription was performed with a DNA primer containing the linkers specific for Illumina sequencing and carbon spacers (Integrated DNA Technologies) and an enzyme PrimeScript (Takara Bio). The resulting single-stranded DNA was circularized with DNA CircLigase (Epicentre), and used as a template for PCR. Eleven cycles of PCR were performed with an enzyme PrimeSTAR Max (Takara Bio), which produced the double-stranded DNA ready for sequencing with the Illumina platform. Quantification of the cDNAs and Illumina sequencing were performed as described previously [25], except that a GAIIx single-end run with 36 bp length was employed.

\section{Data analysis}

The fastq files of 36-bp sequenced reads were generated with CASAVA v1.8 (Illumina). For the bulk RNET-seq analysis, the specific adapter sequences were trimmed with Trimmomatic v.0.25 [88] to obtain reads $\geq 21 \mathrm{nt}$ from the $5^{\prime}$ end. The reads $\geq 21$ nt were mapped to the reference genome of $E$. coli $\mathrm{K}-12$ strain W3110 (NC_007779.1) [or MG1665 (NC_000913.2) when transcription start site information was necessary for the sequence analysis], using Bowtie2 v.2.1.0 with default parameters [89]. We verified high Phred quality scores $Q$ through the mapped reads in both WT and $\triangle$ gre $A B$ strains (Fig. S3 in Additional file 1; $Q_{\text {median }}=38$ and $Q_{\text {lowest }}>28$ ) [90]. The RNA read sequences were not always uniquely mapped and could be found at multiple locations on the E. coli genome. We arbitrarily included reads that were mapped to multiple chromosomal locations ( $40 \%$ of the total mapped reads for the bulk RNET-seq analysis) because both their inclusion and exclusion would generate artifacts. We showed that such multiple mapping events were highly enriched in rRNA genes and IS elements (Fig. S7 in Additional file 1). To analyze RNAP pausing on the E. coli chromosome, we counted the number of reads at every genomic nucleotide position using the mpileup command of SAM tools v.0.1.18 with $-\mathrm{A}-\mathrm{B}$ parameters [91]. Pausing sites were defined as described in the main text and the legend of Fig. 3A. The information contents of PIEs were visualized using WebLogo [51, 92]. The bulk RNET-seq also found a single rRNA sequence, which was the same for both WT and $\triangle$ gre $A B$ cells. Its sequence was completely different from either PIE determined for mRNA genes (Fig. 3A; Fig. S7 in Additional file 1). The sequencing reads for this RNA also had an extraordinarily high $\left(\sim 3 \times 10^{6}\right)$ read depth, suggesting presence of a very strong pause at this location or, more likely, formation of a stable binary complex of RNAP with this rRNA fragment in vivo or during the RNase treatment. 
For single-length RNET-seq analyses, all sequence reads were sorted by their length into $6 \mathrm{nt}$ to $30 \mathrm{nt}$. The reads were mapped to the reference sequence (NC_007779.1) as described above. The distribution of each read length is shown in Fig. 1B. In the PIEs for 14- to 19-nt reads, single-length RNET-seq analysis found an enrichment of $G$ residues at the 3' cleavage site generated by RNases used for the 5' RNA trimming (Figs. S9 and S10 in Additional file 1). Because RNaseT1 specifically cuts GpN bonds in the RNA, whereas RNaseA cuts $\mathrm{CpN}$ or UpN bonds, a large fraction of RNA species of 14-19 nt had been cleaved at GpN, the signature of an RNaseT1 cut (Figs. S9 and S10 in Additional file 1). This indicates that after the RNase treatment for ECs, the canonical assignment where 14-nt RNA corresponds to the post-translocated state, was made mostly when a G residue was positioned immediately $5^{\prime}$ of the nascent RNA protected by RNAP. Consequently, this bias underestimated the presence in vivo of the post-translocated state (14 nt) primarily and that of the pre-translocated state (15 nt) secondly as opposed to the backtracked states (>15 nt). For this reason, 14- to 18-nt reads were used for obtaining information about translocated/backtracked states in paused RNAP in vivo, and $\geq 21$-nt reads lacking an obvious signature of the RNaseT1 cut (no signature of translocated/backtracked states) were used for a genomewide analysis of pausing pattern.

The sequence reads for each gene were determined along the length of the gene using the HTSeq v.0.5.4p2 [93]. A gene annotation file of E. coli W3110 was downloaded from the ftp server of Ensembl [94]. To accommodate the seven nearly identical rRNA genes, we divided the read depths found in rRNA genes by seven to represent the average of one rRNA operon. Normalization of read counts for each gene or each gene body in the bulk RNET-seq analysis was performed by $10^{6} \times(\mathrm{Sum}$ of reads)/Total mapped reads (equal to 6,967,786 for WT or $11,174,399$ for $\triangle$ gre $A B$ ). The transcription start site and operon data sets in $E$. coli were downloaded from RegulonDB [95]. For the transcription start site, we used positions having the maximum transcription start site frequency in the high-throughput transcription initiation mapping (version 3.0). The error rate per read position was calculated by counting each type of error in each read position using CIGAR and MD:Z tags of SAM format with a Perl script. All the Perl scripts developed and used in this study are publicly available at [96].

\section{Data availability}

Raw sequencing data and processed data are available at the Gene Expression Omnibus under accession number GSE62102 [97].

\section{Additional files}

\begin{abstract}
Additional file 1: Figure S1. A Purified RNA used for the RNET-seq analysis in E. coli WT and $\triangle$ greAB cells. B Elongation activities of the purified initiation/elongation complexes with and without Gre proteins (see "Materials and methods" for details). C Quantification of the gel images shown in Fig. S1B. Figure S2. Short reads derive from initiation complexes. The information about the transcription start sites was obtained as described in Materials and methods. Figure S3. Phred quality scores per position through 21-nt reads. Figure S4. A Genome-wide gene-specific comparisons of the bulk RNET-seq results between E. coli WT and $\triangle$ gre $A B$ cells. B Gre-dependent suppression of pausing in the Up region is correlated with 5' UTR lengths close to 50 bases. Figure S5. Cartoon images for pausing and non pausing when defined by $P(0.9$, $100)$ or $P(0.9,160)$. The latter is shown in parentheses. Figure S6. A The PIEs for WT and $\triangle$ greAB strains (Fig. $3 \mathrm{~A}$ ) are unique. B The PIEs identified by bulk RNET-seq are robust against parameters $\phi$ and $\delta$. Figure S7. Mapping quality (mapq) controls of the pause sites in WT (top) and $\triangle$ gre $A B$ (bottom) cells. The mapq is defined as $-10 \log _{10} p$, where $p$ is an estimate of the probability that the read mapped to a position(s) in addition to the particular position [57]. Figure S8. A Scatter plot of antisense and sense transcription in WT strain. B Antisense transcriptional pauses observed in the ins $A B$ genes of WT cells. Figure S9. PIEs determined by single-length RNET-seq in WT strain. Figure S10. PIEs determined by single-length RNET-seq in $\triangle$ greAB strain. Figure S11. A Box plots of mapq $_{\text {mean }}$ for the pause sites identified by single-length RNET-seq analysis using 14-, 15- or 16-nt reads. B G G $_{-9}$ is favored for the pause sites with low mapping quality in 14-nt reads. Figure S12 Effect of 5-methyl-dC $C_{+1}$ in the template DNA on RNAP translocation in vitro. Figure S13. Error rates per position in nascent transcripts detected by single-length (14-18 nt) RNET-seq.
\end{abstract}

Additional file 2: Table S1. Frequency matrix, position weight matrix and MAP scores for PIEs. Table S2. MAP scores for transcription factors in E. coli.

Additional file 3: Method S1. Calculation of MAP scores for PIEs. Method S2. Inactivation of greA and greB genes.

\section{Abbreviations}

bp: base pair; CDS: coding DNA sequence; EC: elongation complex; IS: insertion sequence; NET-seq: native elongating transcript sequencing; nt: nucleotide; NTP: ribonucleoside triphosphate; PIE: pause-inducing element; PPi: pyrophosphate; RNAP: RNA polymerase; RNET-seq: RNase-footprinting followed by NET-seq; UTR: untranslated region; WT: wild type.

\section{Competing interests}

The authors declare that they have no competing interests.

\section{Authors' contributions}

Conceived and designed the experiments: MI, MK and TO. Performed the RNET-seq and biochemical experiments: MI. Performed the recombineering: MB. Analyzed data: MI, HT and CM. Discussed data: MI, MK, TO, HT and DLC. Wrote the paper: MI, MK and DLC. All authors read and approved the final manuscript.

\section{Acknowledgments}

We thank Lucyna Lubkowska for E. coli RNAP and Gre proteins and Maria Kireeva for discussions. We are also grateful to Zachary Burton for discussions at the initial stage of this work and sharing his error analysis data of yeast NET-seq [35]. We also thank the $\mathrm{NCl}$ sequencing facility for Illumina sequencing and support. This work was supported by the Intramural Research Program of the National Institutes of Health, National Cancer Institute, Center for Cancer Research to MK. MI is partially supported by a fellowship from JSPS. HT and TO are supported by MEXT KAKENHI (number 221S0002) and JSPS KAKENHI (number 26450090), respectively.

\section{Author details}

${ }^{1}$ Center for Cancer Research, National Cancer Institute, Frederick, MD 21702, USA. ${ }^{2}$ Medical Mycology Research Center, Chiba University, 1-8-1 Inohana, Chuo-ku, Chiba 260-8673, Japan. ${ }^{3}$ Graduate School of Biological Sciences, Nara Institute of Science and Technology, 8916-5, Ikoma, Nara 630-0192, Japan. 
Received: 13 April 2015 Accepted: 5 May 2015

Published online: 15 May 2015

\section{References}

1. Imashimizu M, Shimamoto N, Oshima T, Kashlev M. Transcription elongation: Heterogeneous tracking of RNA polymerase and its biological implications. Transcription. 2014;5:e28285-28281-28210.

2. Bailey MJ, Hughes C, Koronakis V. RfaH and the ops element, components of a novel system controlling bacterial transcription elongation. Mol Microbiol. 1997;26:845-51.

3. Tomar SK, Artsimovitch I. NusG-Spt5 proteins-Universal tools for transcription modification and communication. Chem Rev. 2013;113:8604-19.

4. Adelman K, Lis JT. Promoter-proximal pausing of RNA polymerase II: emerging roles in metazoans. Nat Rev Genet. 2012;13:720-31.

5. Perdue SA, Roberts JW. Sigma(70)-dependent transcription pausing in Escherichia coli. J Mol Biol. 2011;412:782-92.

6. Yamaguchi $Y$, Shibata $H$, Handa $H$. Transcription elongation factors DSIF and NELF: promoter-proximal pausing and beyond. Biochim Biophys Acta. 2013;1829:98-104

7. Yamaguchi Y, Takagi T, Wada T, Yano K, Furuya A, Sugimoto S, et al. NELF, a multisubunit complex containing RD, cooperates with DSIF to repress RNA polymerase II elongation. Cell. 1999;97:41-51.

8. Rogatsky I, Adelman K. Preparing the first responders: building the inflammatory transcriptome from the ground up. Mol Cell. 2014;54:245-54

9. Nechaev S, Adelman K. Pol II waiting in the starting gates: Regulating the transition from transcription initiation into productive elongation. Biochim Biophys Acta. 2011;1809:34-45.

10. Landick $R$. The regulatory roles and mechanism of transcriptional pausing. Biochem Soc Trans. 2006;34:1062-6.

11. Nudler E. RNA polymerase active center: the molecular engine of transcription. Annu Rev Biochem. 2009;78:335-61.

12. Imashimizu M, Kireeva ML, Lubkowska L, Gotte D, Parks AR, Strathern JN, et al. Intrinsic translocation barrier as an initial step in pausing by RNA polymerase II. J Mol Biol. 2013;425:697-712.

13. Zhou J, Ha KS, La Porta A, Landick R, Block SM. Applied force provides insight into transcriptional pausing and its modulation by transcription factor NusA. Mol Cell. 2011:44:635-46.

14. Yakhnin AV, Yakhnin $H$, Babitzke P. Function of the Bacillus subtilis transcription elongation factor NusG in hairpin-dependent RNA polymerase pausing in the trp leader. Proc Natl Acad Sci U S A. 2008;105:16131-6.

15. Vitiello CL, Kireeva ML, Lubkowska L, Kashlev M, Gottesman M. Coliphage HK022 Nun protein inhibits RNA polymerase translocation. Proc Natl Acad Sci U S A. 2014;111:E2368-75.

16. Toulokhonov I, Landick R. The flap domain is required for pause RNA hairpin inhibition of catalysis by RNA polymerase and can modulate intrinsic termination. Mol Cell. 2003;12:1125-36.

17. Weixlbaumer A, Leon K, Landick R, Darst SA. Structural basis of transcriptional pausing in bacteria. Cell. 2013;152:431-41.

18. Artsimovitch I, Landick R. Pausing by bacterial RNA polymerase is mediated by mechanistically distinct classes of signals. Proc Natl Acad Sci U S A. 2000;97:7090-5.

19. Neuman KC, Abbondanzieri EA, Landick R, Gelles J, Block SM. Ubiquitous transcriptional pausing is independent of RNA polymerase backtracking. Cell. 2003:115:437-47.

20. Komissarova N, Kashlev M. RNA polymerase switches between inactivated and activated states By translocating back and forth along the DNA and the RNA. J Biol Chem. 1997;272:15329-38.

21. Dutta D, Shatalin K, Epshtein V, Gottesman ME, Nudler E. Linking RNA polymerase backtracking to genome instability in E. coli. Cell. 2011;146:533-43.

22. Nudler E. RNA polymerase backtracking in gene regulation and genome instability. Cell. 2012;149:1438-45.

23. Aguilera A, Garcia-Muse T. R loops: from transcription byproducts to threats to genome stability. Mol Cell. 2012;46:115-24.

24. Helmrich A, Ballarino M, Nudler E, Tora L. Transcription-replication encounters, consequences and genomic instability. Nat Struct Mol Biol. 2013:20:412-8.

25. Imashimizu M, Oshima T, Lubkowska L, Kashlev M. Direct assessment of transcription fidelity by high-resolution RNA sequencing. Nucleic Acids Res. 2013:41:9090-104.
26. Gordon AJ, Halliday JA, Blankschien MD, Burns PA, Yatagai F, Herman C. Transcriptional infidelity promotes heritable phenotypic change in a bistable gene network. PLoS Biol. 2009;7:e44

27. Goldsmith M, Tawfik DS. Potential role of phenotypic mutations in the evolution of protein expression and stability. Proc Natl Acad Sci U S A. 2009;106:6197-202.

28. Paoloni-Giacobino A, Rossier C, Papasavvas MP, Antonarakis SE. Frequency of replication/transcription errors in $(A) /(T)$ runs of human genes. Hum Genet. 2001;109:40-7.

29. Rodin SN, Rodin AS, Juhasz A, Holmquist GP. Cancerous hyper-mutagenesis in p53 genes is possibly associated with transcriptional bypass of DNA lesions. Mutat Res. 2002;510:153-68.

30. Hubbard K, Catalano J, Puri RK, Gnatt A. Knockdown of TFIIS by RNA silencing inhibits cancer cell proliferation and induces apoptosis. BMC Cancer. 2008:8:133.

31. Borukhov S, Sagitov V, Goldfarb A. Transcript cleavage factors from E coli. Cell. 1993;72:459-66.

32. Fish RN, Kane CM. Promoting elongation with transcript cleavage stimulatory factors. Biochim Biophys Acta. 2002;1577:287-307.

33. Kireeva ML, Kashlev M. Mechanism of sequence-specific pausing of bacterial RNA polymerase. Proc Natl Acad Sci U S A. 2009;106:8900-5.

34. Bai L, Shundrovsky A, Wang MD. Sequence-dependent kinetic model for transcription elongation by RNA polymerase. J Mol Biol. 2004;344:335-49.

35. Churchman LS, Weissman JS. Nascent transcript sequencing visualizes transcription at nucleotide resolution. Nature. 2011;469:368-73.

36. Komissarova N, Kashlev M. Functional topography of nascent RNA in elongation intermediates of RNA polymerase. Proc Natl Acad Sci U S A. 1998:95:14699-704

37. Walter G, Zillig W, Palm P, Fuchs E. Initiation of DNA-dependent RNA synthesis and the effect of heparin on RNA polymerase. Eur J Biochem. 1967;3:194-201.

38. Kashlev M, Nudler E, Severinov K, Borukhov S, Komissarova N, Goldfarb A. Histidine-tagged RNA polymerase of Escherichia coli and transcription in solid phase. Methods Enzymol. 1996;274:326-34.

39. Shimamoto N. Nanobiology of RNA polymerase: biological consequence of inhomogeneity in reactant. Chem Rev. 2013;113:8400-22

40. Mooney RA, Davis SE, Peters JM, Rowland JL, Ansari AZ, Landick R. Regulator trafficking on bacterial transcription units in vivo. Mol Cell. 2009;33:97-108.

41. Giannoukos G, Ciulla DM, Huang K, Haas BJ, Izard J, Levin JZ, et al. Efficient and robust RNA-seq process for cultured bacteria and complex community transcriptomes. Genome Biol. 2012;13:R23.

42. Epshtein V, Nudler E. Cooperation between RNA polymerase molecules in transcription elongation. Science. 2003;300:801-5.

43. Zellars $\mathrm{M}$, Squires $\mathrm{CL}$. Antiterminator-dependent modulation of transcription elongation rates by NusB and NusG. Mol Microbiol. 1999;32:1296-304.

44. Herbert KM, Zhou J, Mooney RA, Porta AL, Landick R, Block SM. E. coli NusG inhibits backtracking and accelerates pause-free transcription by promoting forward translocation of RNA polymerase. J Mol Biol. 2010;399:17-30

45. Sen $R$, Nagai $H$, Shimamoto N. Conformational switching of Escherichia coli RNA polymerase-promoter binary complex is facilitated by elongation factor GreA and GreB. Genes Cells. 2001;6:389-401.

46. Stepanova E, Lee J, Ozerova M, Semenova E, Datsenko K, Wanner BL, et al. Analysis of promoter targets for Escherichia coli transcription elongation factor GreA in vivo and in vitro. J Bacteriol. 2007;189:8772-85.

47. Gordon AJ, Satory D, Halliday JA, Herman C. Heritable change caused by transient transcription errors. PLoS Genet. 2013;9:e1003595.

48. Susa M, Kubori T, Shimamoto N. A pathway branching in transcription initiation in Escherichia coli. Mol Microbiol. 2006;59:1807-17.

49. Cho BK, Zengler K, Qiu Y, Park YS, Knight EM, Barrett CL, et al. The transcription unit architecture of the Escherichia coli genome. Nat Biotechnol. 2009:27:1043-9.

50. Proshkin S, Rahmouni AR, Mironov A, Nudler E. Cooperation between translating ribosomes and RNA polymerase in transcription elongation. Science. 2010;328:504-8.

51. Schneider TD, Stephens RM. Sequence logos: a new way to display consensus sequences. Nucleic Acids Res. 1990;18:6097-100.

52. Hertz GZ, Hartzell 3rd GW, Stormo GD. Identification of consensus patterns in unaligned DNA sequences known to be functionally related. Comput Appl Biosci. 1990;6:81-92.

53. Liu XS, Brutlag DL, Liu JS. An algorithm for finding protein-DNA binding sites with applications to chromatin-immunoprecipitation microarray experiments. Nat Biotechnol. 2002;20:835-9. 
54. Vassylyev DG, Vassylyeva MN, Perederina A, Tahirov TH, Artsimovitch I. Structural basis for transcription elongation by bacterial RNA polymerase. Nature. 2007;448:157-62.

55. Larson MH, Mooney RA, Peters JM, Windgassen T, Nayak D, Gross CA, et al. A pause sequence enriched at translation start sites drives transcription dynamics in vivo. Science. 2014;344:1042-7.

56. Komissarova N, Becker J, Solter S, Kireeva M, Kashlev M. Shortening of RNA: DNA hybrid in the elongation complex of RNA polymerase is a prerequisite for transcription termination. Mol Cell. 2002;10:1151-62.

57. Li H, Ruan J, Durbin R. Mapping short DNA sequencing reads and calling variants using mapping quality scores. Genome Res. 2008;18:1851-8.

58. Wei W, Pelechano V, Jarvelin Al, Steinmetz LM. Functional consequences of bidirectional promoters. Trends Genet. 2011;27:267-76.

59. Peters JM, Mooney RA, Kuan PF, Rowland JL, Keles S, Landick R. Rho directs widespread termination of intragenic and stable RNA transcription. Proc Natl Acad Sci U S A. 2009;106:15406-11.

60. Dornenburg JE, Devita AM, Palumbo MJ, Wade JT. Widespread antisense transcription in Escherichia coli. mBio. 2010;1:e00024-10.

61. Stolt $\mathrm{P}$, Zillig W. Antisense RNA mediates transcriptional processing in an archaebacterium, indicating a novel kind of RNase activity. Mol Microbiol. 1993;7:875-82.

62. Wexler M, Sargent F, Jack RL, Stanley NR, Bogsch EG, Robinson C, et al. TatD is a cytoplasmic protein with DNase activity. No requirement for TatD family proteins in sec-independent protein export. J Biol Chem. 2000;275:16717-22.

63. Bittner M, Saldias S, Estevez C, Zaldivar M, Marolda CL, Valvano MA, et al. O-antigen expression in Salmonella enterica serovar Typhi is regulated by nitrogen availability through $\mathrm{RpoN}$-mediated transcriptional control of the rfaH gene. Microbiology. 2002;148:3789-99.

64. Sekine $Y$, Ohtsubo E. Frameshifting is required for production of the transposase encoded by insertion sequence 1. Proc Natl Acad Sci U S A. 1989;86:4609-13.

65. Xuan JC, Weber IT. Crystal structure of a B-DNA dodecamer containing inosine, d(CGCIAATTCGCG), at 2.4 A resolution and its comparison with other B-DNA dodecamers. Nucleic Acids Res. 1992;20:5457-64

66. Hawryluk PJ, Ujvari A, Luse DS. Characterization of a novel RNA polymerase II arrest site which lacks a weak 3' RNA-DNA hybrid. Nucleic Acids Res. 2004;32:1904-16.

67. Herbert KM, La Porta A, Wong BJ, Mooney RA, Neuman KC, Landick R, et al. Sequence-resolved detection of pausing by single RNA polymerase molecules. Cell. 2006;125:1083-94.

68. Imashimizu M, Kashlev M. Unveiling translocation intermediates of RNA polymerase. Proc Natl Acad Sci U S A. 2014;111:7507-8.

69. Bertrand $\mathrm{H}, \mathrm{Ha}$-Duong $\mathrm{T}$, Fermandjian S, Hartmann B. Flexibility of the B-DNA backbone: effects of local and neighbouring sequences on pyrimidine-purine steps. Nucleic Acids Res. 1998;26:1261-7.

70. Grokhovsky SL, I'icheva IA, Nechipurenko DY, Golovkin MV, Panchenko LA, Polozov RV, et al. Sequence-specific ultrasonic cleavage of DNA. Biophys J. 2011;100:117-25.

71. Nikolova EN, Bascom GD, Andricioaei I, Al-Hashimi HM. Probing sequence-specific DNA flexibility in a-tracts and pyrimidine-purine steps by nuclear magnetic resonance (13)C relaxation and molecular dynamics simulations. Biochemistry. 2012;51:8654-64.

72. Jonkers I, Kwak H, Lis JT. Genome-wide dynamics of Pol II elongation and its interplay with promoter proximal pausing, chromatin, and exons. eLife. 2014;3:e02407.

73. Meints GA, Drobny GP. Dynamic impact of methylation at the M. Hhai target site: a solid-state deuterium NMR study. Biochemistry. 2001:40:12436-43.

74. Geahigan KB, Meints GA, Hatcher ME, Orban J, Drobny GP. The dynamic impact of CpG methylation in DNA. Biochemistry. 2000;39:4939-46.

75. Kashlev M, Nudler E, Goldfarb A, White T, Kutter E. Bacteriophage T4 Alc protein: a transcription termination factor sensing local modification of DNA. Cell. 1993;75:147-54.

76. Walmaca C, Cheung AC, Kireeva ML, Lubkowska L, Ye C, Gotte D, et al. Mechanism of translesion transcription by RNA polymerase $\|$ and its role in cellular resistance to DNA damage. Mol Cell. 2012;46:1-12.

77. Kent T, Kashkina E, Anikin M, Temiakov D. Maintenance of RNA-DNA hybrid length in bacterial RNA polymerases. J Biol Chem. 2009;284:13497-504.

78. Yager TD, von Hippel PH. A thermodynamic analysis of RNA transcript elongation and termination in Escherichia coli. Biochemistry. 1991;30:1097-118.
79. Keene RG, Mueller A, Landick R, London L. Transcriptional pause, arrest and termination sites for RNA polymerase II in mammalian $\mathrm{N}$ - and c-myc genes. Nucleic Acids Res. 1999;27:3173-82.

80. Reeder TC, Hawley DK. Promoter proximal sequences modulate RNA polymerase II elongation by a novel mechanism. Cell. 1996;87:767-77.

81. Vvedenskaya IO, Vahedian-Movahed H, Bird JG, Knoblauch JG, Goldman SR, Zhang Y, et al. Transcription. Interactions between RNA polymerase and the "core recognition element" counteract pausing. Science. 2014;344:1285-9.

82. Deaton AM, Bird A. CpG islands and the regulation of transcription. Genes Dev. 2011;25:1010-22.

83. Tehranchi AK, Blankschien MD, Zhang Y, Halliday JA, Srivatsan A, Peng J, et al. The transcription factor DksA prevents conflicts between DNA replication and transcription machinery. Cell. 2010;141:595-605.

84. Poteete AR. Recombination phenotypes of Escherichia coli greA mutants BMC Mol Biol. 2011;12:12.

85. Ginno PA, Lott PL, Christensen HC, Korf I, Chedin F. R-loop formation is a distinctive characteristic of unmethylated human $\mathrm{CpG}$ island promoters. Mol Cell. 2012:45:814-25

86. Jinks-Robertson S, Bhagwat AS. Transcription-associated mutagenesis. Annu Rev Genet. 2014;48:341-59.

87. Churchman LS, Weissman JS. Native elongating transcript sequencing (NET-seq). Curr Protoc Mol Biol. 2012;4:Unit 4 14 11-17.

88. Lohse M, Bolger AM, Nagel A, Fernie AR, Lunn JE, Stitt M, et al. RobiNA: a user-friendly, integrated software solution for RNA-Seq-based transcriptomics. Nucleic Acids Res. 2012;40:W622-7.

89. Langmead B, Salzberg SL. Fast gapped-read alignment with Bowtie 2. Nat Methods. 2012;9:357-9

90. Ewing B, Green P. Base-calling of automated sequencer traces using phred II. Error probabilities. Genome Res. 1998;8:186-94.

91. Li H, Handsaker B, Wysoker A, Fennell T, Ruan J, Homer N, et al. The Sequence Alignment/Map format and SAMtools. Bioinformatics. 2009;25:2078-9.

92. Crooks GE, Hon G, Chandonia JM, Brenner SE. WebLogo: a sequence logo generator. Genome Res. 2004;14:1188-90.

93. Anders S, Pyl PT, Huber W. HTSeq-a Python framework to work with high-throughput sequencing data. Bioinformatics. 2015;31:166-9.

94. EnsemblBacteria. http://bacteria.ensembl.org/info/data/ftp/index.html.

95. Salgado H, Peralta-Gil M, Gama-Castro S, Santos-Zavaleta A, Muniz-Rascado L, Garcia-Sotelo JS, et al. RegulonDB v8.0: omics data sets, evolutionary conservation, regulatory phrases, cross-validated gold standards and more. Nucleic Acids Res. 2013:41:D203-13.

96. Perl scripts. https://github.com/kuu774/net-seq_analysis

97. Gene Expression Omnibus: Series GSE62102. http://www.ncbi.n/m.nih.gov/ geo/query/acc.cgi?acc=GSE62102

\section{Submit your next manuscript to BioMed Central and take full advantage of:}

- Convenient online submission

- Thorough peer review

- No space constraints or color figure charges

- Immediate publication on acceptance

- Inclusion in PubMed, CAS, Scopus and Google Scholar

- Research which is freely available for redistribution 